\title{
Existence of conformal metrics with prescribed scalar curvature on the four dimensional half sphere
}

\author{
Mohamed Ben Ayed, Rabeh Ghoudi and Kamal Ould Bouh
}

\begin{abstract}
In this paper, we consider the problem of prescribing the scalar curvature under minimal boundary conditions on the four dimensional half sphere. Using dynamical and topological methods involving the study of the critical points at infinity of the associated variational structure, we prove some existence results like Bahri-Coron theorem. Furthermore, we consider the approximate subcritical problem and we construct some solutions which blow up at two different points, one of them lay on the boundary and the other one is an interior point.
\end{abstract}

Mathematics Subject Classification (2000). 35J20, 35J60.

Keywords. Variational problem, Scalar curvature, Critical points at infinity.

\section{Introduction}

In this paper we study some nonlinear problem arising from conformal geometry. Precisely, let $\left(M^{n}, g\right)$ be a $n$-dimensional Riemannian manifold with boundary, $n \geq 3$ and let $\tilde{g}=u^{4 /(n-2)} g$, be a conformal metric to $g$, where $u$ is a smooth positive function, then the following equations relate the scalar curvatures $R_{g}, R_{\tilde{g}}$ and the mean curvatures of the boundary $h_{g}, h_{\tilde{g}}$, with respect to $g$ and $\tilde{g}$ respectively.

$$
\begin{cases}-c_{n} \Delta_{g} u+R_{g} u=R_{\tilde{g}} u^{(n+2) /(n-2)} & \text { in } M, \\ \frac{2}{n-2} \frac{\partial u}{\partial \nu}+h_{g} u=h_{\tilde{g}} u^{n /(n-2)} & \text { on } \partial M,\end{cases}
$$

where $c_{n}=4(n-1) /(n-2)$ and $\nu$ denotes the outward normal vector with respect to $g$.

K. Ould Bouh is supported by TWAS Fellowships for Research and Advanced Training Programme. 
In view of the above equations, the following problem naturally arises: given two functions $K: M \rightarrow \mathbb{R}$ and $H: \partial M \rightarrow \mathbb{R}$, does there exist a metric $\tilde{g}$ conformally equivalent to $g$ such that $R_{\tilde{g}}=K$ and $h_{\tilde{g}}=H$ ? According to equation (1), the problem is equivalent to finding a smooth positive solution $u$ of the following equation

$$
\begin{cases}-c_{n} \Delta_{g} u+R_{g} u=K u^{(n+2) /(n-2)} & \text { in } M, \\ \frac{2}{n-2} \frac{\partial u}{\partial \nu}+h_{g} u=H u^{n /(n-2)} & \text { on } \partial M .\end{cases}
$$

Such a problem was studied in $[1,9,10,14-18,21]$ and the references therein. In this paper, we consider the case of the standard four dimensional half sphere under minimal boundary conditions. More precisely, let $K$ be a $C^{3}$ positive Morse function on $\overline{S_{+}^{4}}$, we look for conditions on $K$ to ensure the existence of a positive solution of the problem

$$
\begin{cases}L_{g} u:=-\Delta_{g} u+2 u=K u^{3}, \quad u>0 & \text { in } S_{+}^{4} \\ \partial u / \partial \nu=0 & \text { on } \partial S_{+}^{4},\end{cases}
$$

where $g$ is the standard metric of $S_{+}^{n}=\left\{x \in \mathbb{R}^{n+1} /|x|=1, x_{n+1}>0\right\}$.

Li [21], and Djadli-Malchiodi-Ould Ahmedou [15] studied this problem when the manifold is the three dimensional standard half sphere. Their approach involves a fine blow up analysis of some subcritical approximations and the use of the topological degree tools. Ben Ayed-El Mehdi-Ould Ahmedou [9], [10] gave some topological conditions on $K$ to prescribe the scalar curvature under minimal boundary conditions on half spheres of dimension $n \geq 4$.

Note that problem $(P)$ is in some sense related to the well known scalar curvature problem

$$
-\Delta_{g} u+(n(n-2) / 4) u=K u^{(n+2) /(n-2)}, \quad u>0 \quad \text { in } S^{n}
$$

to which much work has been devoted (see $[3,4,7,13,14,19-21]$ and the references therein).

As for (3), there are topological obstructions of Kazdan-Warner type to solve $(P)$ (see $[11])$ and so a natural question arises: under which conditions on $K$, $(P)$ has a positive solution?

Regarding problem (3), Bahri [3] observed that a new phenomenon appears in dimension $n \geq 5$ due to the fact that the self interaction of the functions failing the Palais-Smale condition dominates the interaction of two of those functions. In the three dimension case, the reverse happens (see [4]). In the four dimensional case, we have a balance phenomenon, that is, the self interaction and the interaction are of the same size (see [7]).

In the case of $(P)$, Ben Ayed et al. proved that the balance phenomenon appears if we assume that $(\partial K / \partial \nu)(y)<0$ where $y$ is any critical point of $K_{1}=K_{\mid \partial S_{+}^{4}}$. Moreover, it is proved that this phenomenon appears also when the manifold is the three dimensional half sphere (see [15]). 
Note that the embedding of $H^{1}\left(S_{+}^{4}\right)$ into $L^{4}\left(S_{+}^{4}\right)$ is noncompact. Hence, for the study of problem $(P)$, it is interesting to approach it by a family of subcritical problems $\left(P_{\varepsilon}\right)$

$$
\begin{cases}-\Delta_{g} u+2 u=K u^{3-\varepsilon}, \quad u>0 & \text { in } S_{+}^{4} \\ \partial u / \partial \nu=0 & \text { on } \partial S_{+}^{4},\end{cases}
$$

and we need to study the asymptotic behavior of the solutions $\left(u_{\varepsilon}\right)$ as $\varepsilon \rightarrow 0$. Observe that, since $\varepsilon>0$, problem $\left(P_{\varepsilon}\right)$ has always a positive solution $\left(u_{\varepsilon}\right)$. It is well known that, for the minimizing solutions, $\left(u_{\varepsilon}\right)$ has to converge to a solution of $(P)$ or to blow up at one point which is the maximum of $K$ or of $K_{1}$. For the other solutions $\left(u_{\varepsilon}\right)$ (not minimizing solutions) and in the case of the three dimensional half sphere, Djadli et al. [15] proved that $\left(u_{\varepsilon}\right)$ can blow up at $\left(x_{1}, \ldots, x_{p}\right)$ where the points $x_{i}$ 's are different critical points of $K_{1}$ with $(\partial K / \partial \nu)\left(x_{i}\right)>0$. Furthermore they proved that the $x_{i}$ 's are isolated simple blow ups (see [19,20] for the definition) which implies that, writing $u_{\varepsilon}=\sum_{i \leq p} \alpha_{i}^{\varepsilon} \delta_{\left(a_{i}^{\varepsilon}, \lambda_{i}^{\varepsilon}\right)}+v_{\varepsilon}$, we have that $\left|a_{i}-a_{j}\right| \geq c>0$ for $i \neq j$ (the function $\bar{\delta}_{(a, \lambda)}$ is defined in (1.1)). Hence, the tower bubble solutions do not exist. Moreover Ben Ayed et al. [10] proved that there are critical points at infinity (following the terminology of A. Bahri) for the functional $J_{0}$ (defined by (1.2)) associated to the problem $(P)$.

Recall that the critical point at infinity is the limit of the orbits of the gradient flow of $J_{0}$ which remain in $V(p, \eta(s))$, where $\eta(s)$ is a given function which tends to 0 as $s$ tends to infinity and $V(p, \eta)$ is defined by (3.1) below (for more details, see Definition 4.1 below). In other words, this implies the existence of solutions $\left(u_{\varepsilon}\right)$ which blow up at $\left(y_{1}, \ldots, y_{p}\right)$ where the points $y_{i}$ 's are different critical points of $K$ in $S_{+}^{4}$. Note that, in [10], the authors assumed that $(\partial K / \partial \nu)<0$ at each critical point of $K_{1}$. This assumption allows them to cancel the influence of the boundary. However, up to the knowledge of the authors, there is no result about the solutions which blow up at a mixed type of the critical points of $K$ and $K_{1}$ i.e. critical point $z$ of $K_{1}$ with $(\partial K / \partial \nu)(z)>0$ and critical point $y$ of $K$.

In this paper, we generalize the results of [10]. We cancel the assumption of [10] which is $(\partial K / \partial \nu)<0$ at each critical point of $K_{1}$ that is the function $K_{1}=K_{\mid \partial S_{+}^{4}}$ can have some critical points with $(\partial K / \partial \nu)>0$. Our problem becomes more difficult, we need to understand the influence of the boundary in the characterization of the critical points at infinity.

The goal of our first result is the construction of some solutions $\left(u_{\varepsilon}\right)$ of $\left(P_{\varepsilon}\right)$ which blow up at two different points, one of them lies on the boundary and the other is an interior point. This result implies that there are more critical points at infinity than the authors found in [10].

Before stating the result, we need to introduce some notations. For $a \in \overline{S_{+}^{4}}$ and $\lambda>0$, let

$$
\delta_{(a, \lambda)}(x)=c_{0} \frac{\lambda}{\left(\lambda^{2}+1+\left(1-\lambda^{2}\right) \cos d(a, x)\right)}
$$


where $d$ is the geodesic distance on $\left(\overline{S_{+}^{4}}, g\right)$ and $c_{0}$ is chosen so that $\delta_{(a, \lambda)}$ is a solution of

$$
-\Delta u+2 u=u^{3}, \quad u>0, \quad \text { in } S^{4} .
$$

We denote by $P \delta_{(a, \lambda)}$ the projection of the function $\delta_{(a, \lambda)}$ defined by

$$
\begin{gathered}
-\Delta P \delta_{(a, \lambda)}+2 P \delta_{(a, \lambda)}=-\Delta \delta_{(a, \lambda)}+2 \delta_{(a, \lambda)}, \quad \text { in } S_{+}^{4}, \\
\partial P \delta_{(a, \lambda)} / \partial \nu=0 \text { on } \partial S_{+}^{4} .
\end{gathered}
$$

Let $G$ be the Green's function of $L_{g}:=-\Delta+2 I d$ on $S_{+}^{4}$ and $H$ be its regular part defined by

$$
\left\{\begin{array}{l}
G(x, y)=(1-\cos (d(x, y)))^{-1}+H(x, y), \\
L_{g} H=0 \text { in } S_{+}^{4} ; \quad \partial G / \partial \nu=0 \text { on } \partial S_{+}^{4} .
\end{array}\right.
$$

It is known that $H$ is a positive function and $H(x, x) \rightarrow+\infty$ as $x$ goes to the boundary.

The space $H^{1}\left(S_{+}^{4}\right)$ is equipped with the norm $\|$.$\| and the inner product \langle.,$. defined by

$$
\begin{aligned}
& \|u\|^{2}=\int_{S_{+}^{4}}|\nabla u|^{2}+2 \int_{S_{+}^{4}} u^{2}, \quad \text { and } \\
& \langle u, v\rangle=\int_{S_{+}^{4}} \nabla u \nabla v+2 \int_{S_{+}^{4}} u v, \quad u, v \in H^{1}\left(S_{+}^{4}\right) .
\end{aligned}
$$

Theorem 1.1. Let $z$ be a nondegenerate critical point of $K_{1}=K_{\mid \partial S_{+}^{4}}$ with $(\partial K / \partial \nu)(z)>0$ and $y$ be a nondegenerate critical point of $K$ with $(-\Delta K(y) / 3 K(y)-4 H(y, y))>0$. Then, there exists $\varepsilon_{0}>0$ such that for each $\varepsilon \in\left(0, \varepsilon_{0}\right)$, problem $\left(P_{\varepsilon}\right)$ has a solution $\left(u_{\varepsilon}\right)$ of the form

$$
\begin{gathered}
u_{\varepsilon}=\alpha_{1}^{\varepsilon} \delta_{\left(x_{1}^{\varepsilon}, \lambda_{1}^{\varepsilon}\right)}+\alpha_{2}^{\varepsilon} P \delta_{\left(x_{2}^{\varepsilon}, \lambda_{2}^{\varepsilon}\right)}+v_{\varepsilon}, \quad \text { with } \\
\alpha_{1}^{\varepsilon} \rightarrow K(z)^{-1 / 2} ; \alpha_{2}^{\varepsilon} \rightarrow K(y)^{-1 / 2} ;\left\|v_{\varepsilon}\right\| \rightarrow 0 ; x_{1}^{\varepsilon} \rightarrow z ; x_{2}^{\varepsilon} \rightarrow y, \\
x_{1}^{\varepsilon} \in \partial S_{+}^{4} ; \lambda_{1}^{\varepsilon} \rightarrow+\infty ; \lambda_{1}^{\varepsilon}=c\left(\lambda_{2}^{\varepsilon}\right)^{2}(1+o(1)), \quad \text { as } \varepsilon \rightarrow 0 .
\end{gathered}
$$

Here, $c$ is a positive fixed constant.

In other words, Theorem 1.1 implies that, we have more critical points at infinity (see definition below) than the authors found in [10]. More precisely, for each two critical points $z \in \partial S_{+}^{4}$ with $\partial K / \partial \nu(z)>0$ and $y \in S_{+}^{4}$ with $-\Delta K(y) / 3 K(y)-4 H(y, y)>0$, corresponds a critical point at infinity for the associated variational functional $J_{0}$ (see Proposition 1.3), where

$$
J_{0}(u)=\left(\int_{S_{+}^{4}} K u^{4}\right)^{-1 / 2}, \quad u \in \Sigma^{+}:=\left\{v \in H^{1}\left(S_{+}^{4}\right):\|v\|=1, v>0\right\} .
$$

In the following, we need to characterize all the new critical points at infinity and using the ideas introduced by Bahri [2], we derive some existence results for $(P)$. 
The main difficulty is to understand the location of the concentration points which lie on the boundary. Indeed, the first question which arises is: Do there exist tower bubble solutions for $\left(P_{\varepsilon}\right)$ ? In another sense, is it possible to have two concentration points or more close to each other? This is the aim of Theorem 1.2 below.

In order to state our result, we need to introduce some notations and assumptions.

$\left(\mathbf{H}_{1}\right)$ We assume that $K_{1}=K_{\mid \partial S_{+}^{4}}$ has only nondegenerate critical points $z_{1}, \ldots, z_{r}$ with

$$
\frac{\partial K}{\partial \nu}\left(z_{i}\right)>0, \quad \text { for } 1 \leq i \leq r_{1}, \quad \frac{\partial K}{\partial \nu}\left(z_{i}\right)<0, \quad \text { for } i \geq r_{1}+1 .
$$

We remark that $r_{1}$ can be zero and in this case we are in the same situation than [10].

$\left(\mathbf{H}_{2}\right)$ For each $i \leq r_{1}$, if $z_{i}$ is not a local maximum point, we assume that

$$
\frac{4 c_{3}^{2}}{K_{1}\left(z_{i}\right)}\left(\frac{\partial K}{\partial \nu}\left(z_{i}\right)\right)^{2}<\gamma c_{5} c_{2}^{2}
$$

where $\gamma$ is the least of the absolute values of the eigenvalues of $D^{2} K_{1}\left(z_{i}\right)$ and the $c_{i}$ 's are positive constants defined in the Appendix.

Let $z_{k}$ be a critical point of $K_{1}$ with $\partial K / \partial \nu\left(z_{k}\right)>0$, and for $q \geq 2$, we introduce the set

$$
\begin{aligned}
V(q, \varepsilon, k)= & \left\{\alpha_{1} \delta_{\left(a_{1}, \lambda_{1}\right)}+\cdots+\alpha_{q} \delta_{\left(a_{q}, \lambda_{q}\right)}: a_{i} \in \partial S_{+}^{4}, \lambda_{i}>\varepsilon^{-1},\right. \\
& \alpha_{i}>0 \text { for } i=1, \ldots, q,\left|\alpha_{i}^{2} K\left(a_{i}\right)\left(\alpha_{j}^{2} K\left(a_{j}\right)\right)^{-1}-1\right|<\varepsilon, \\
& \left.\varepsilon_{i j}<\varepsilon \text { and } \forall i a_{i} \in B\left(z_{k}, 2 \mu\right)\right\},
\end{aligned}
$$

where $\varepsilon_{i j}^{-1}=\lambda_{i} / \lambda_{j}+\lambda_{j} / \lambda_{i}+\lambda_{i} \lambda_{j}\left(1-\cos d\left(a_{i}, a_{j}\right)\right) / 2$ and $\varepsilon, \mu$ are small positive parameters.

Theorem 1.2. Assume that $K$ satisfies $\left(H_{1}\right)$ and $\left(H_{2}\right)$. In $V(q, \varepsilon, k)$, there exists a pseudo gradient $Y_{k}$ so that the following holds: There exits a constant $c>0$ independent of $u=\sum_{i=1}^{q} \alpha_{i} \delta_{\left(a_{i}, \lambda_{i}\right)}+v \in V(q, \varepsilon, k)$, so that

$$
\left\langle-\nabla J_{0}(u), Y_{k}\right\rangle \geq c \sum_{i \leq q}\left(\sum_{j \neq i} \varepsilon_{i j}^{3 / 2}+\frac{1}{\lambda_{i}^{3 / 2}}+\left|1-J_{0}(u)^{2} \alpha_{i}^{2} K\left(a_{i}\right)\right|^{3 / 2}\right) .
$$

Furthermore, $\left|Y_{k}\right|$ is bounded and the maximum of the $\lambda_{i}$ 's is not an increasing function along the flow lines of $Y_{k}$ (hence the Palais-Smale condition is satisfied along each flow line in this set).

Note that, Theorem 1.2 implies that, in $V(q, \varepsilon, k)$, there is no critical point and no critical point at infinity. In other words, this result implies that the blow up point $z_{k}$ is an isolated simple blow up point (see [19,20] for the definition) for the problem $\left(P_{\varepsilon}\right)$.

We notice that this result is immediate in the case of the three dimensional half sphere $S_{+}^{3}$. In fact in this case it is clear that $\varepsilon_{i j}=\left(\lambda_{i} / \lambda_{j}+\lambda_{j} / \lambda_{i}+\lambda_{i} \lambda_{j}(1-\right.$ $\left.\left.\cos \left(d\left(a_{i}, a_{j}\right)\right)\right) / 2\right)^{-1 / 2}$ dominates $1 / \lambda_{i}$ where $\lambda_{i}=\max \left(\lambda_{i}, \lambda_{j}\right)$. Hence, (ordering the $\lambda_{i}$ 's : $\lambda_{1} \leq \lambda_{2} \leq \cdots \leq \lambda_{q}$ ) we decrease all the $\lambda_{i}$ 's for $i \geq 2$ and we 
increase $\lambda_{1}$ if it is very small with respect to $\lambda_{2}$. Thus the result follows in this case. In our paper the situation is more delicate since it is possible to get that $\varepsilon_{i j}$ and $\lambda_{i}^{-1}$ are of the same order.

To characterize the critical points at infinity, we need to add some assumptions for the interior critical points of $K$.

$\left(\mathbf{H}_{3}\right)$ All the critical points $y_{i}$ 's of $K$ in $S_{+}^{4}$ satisfy : $\left(-\Delta K\left(y_{i}\right) / 3 K\left(y_{i}\right)\right)-$ $4 H\left(y_{i}, y_{i}\right) \neq 0$.

Without loss of generality, we can assume that $-\Delta K\left(y_{i}\right) / 3 K\left(y_{i}\right)-4 H\left(y_{i}, y_{i}\right)>$ 0 , for $1 \leq i \leq r_{2}$.

For $s \in\left\{1, \ldots, r_{2}\right\}$ and for any s-tuple $\tau_{s}=\left(i_{1}, \ldots, i_{s}\right) \in\left\{1, \ldots, r_{2}\right\}^{s}$ such that $i_{p} \neq i_{q}$ if $p \neq q$, we define a matrix $M\left(\tau_{s}\right)=\left(M_{p q}\right)_{1 \leq p, q \leq s}$, by

$M_{p p}=\frac{-\Delta K\left(y_{i_{p}}\right)}{3 K\left(y_{i_{p}}\right)^{2}}-4 \frac{H\left(y_{i_{p}}, y_{i_{p}}\right)}{K\left(y_{i_{p}}\right)}, \quad M_{p q}=-\frac{4 G\left(y_{i_{p}}, y_{i_{q}}\right)}{\left(K\left(y_{i_{p}}\right) K\left(y_{i_{q}}\right)\right)^{1 / 2}} \quad$ for $p \neq q$,

and we denote by $\rho\left(\tau_{s}\right)$ the least eigenvalue of $M\left(\tau_{s}\right)$.

$\left(\mathbf{H}_{4}\right)$ For any $s \in\left\{1, \ldots, r_{2}\right\}, M\left(\tau_{s}\right)$ is nondegenerate.

Now we are able to give the key point in the theory of the critical points at infinity. Using the notation of A. Bahri (see also Definition 4.1 below), we have the following result.

Proposition 1.3. Assume that $J_{0}$ has no critical points in $\Sigma^{+}$. Then, under the assumptions $\left(H_{1}\right)-\left(H_{4}\right)$, the only critical points at infinity of $J_{0}$ correspond to

$$
\begin{aligned}
& \sum_{i \leq q} K_{1}\left(z_{j_{i}}\right)^{-\frac{1}{2}} \delta_{\left(z_{j_{i}}, \infty\right)}+\sum_{i \leq s} K\left(y_{j_{i}}\right)^{-\frac{1}{2}} P \delta_{\left(y_{j_{i}}, \infty\right)}, \text { with } \\
& \frac{\partial K}{\partial \nu}\left(z_{j_{i}}\right)>0 \text { and } \rho\left(y_{j_{1}}, \ldots, y_{j_{s}}\right)>0
\end{aligned}
$$

where $p \in \mathbb{N}^{*},(q, s) \in \mathbb{N}^{2}$ and $p=q+s$.

Furthermore, such a critical point at infinity has a Morse index equal to

$$
5 s+4 q-1-\sum_{i \leq q} \operatorname{index}\left(K_{1}, z_{j_{i}}\right)-\sum_{i \leq s} \operatorname{index}\left(K, y_{j_{i}}\right) .
$$

Using some topological arguments and some deformation lemmas, Proposition 1.3 implies:

Theorem 1.4. Under the assumptions $\left(H_{1}\right)-\left(H_{4}\right)$, and assume further that $\left(H_{5}\right)$ For each $i \leq r_{1}$, the Morse index of $K_{1}$ at $z_{i}$ is equal to 0 or 2.

$$
\text { If } \quad-1 \neq \sum_{s=1}^{r_{2}} \sum_{\tau_{s}=\left(i_{1}, \ldots, i_{s}\right) / \rho\left(\tau_{s}\right)>0}(-1)^{s-\sum_{j=1}^{s} k_{i_{j}},}
$$

where $k_{i_{j}}:=\operatorname{index}\left(K, y_{i_{j}}\right)$, then problem $(P)$ has a solution.

Note that our result is a generalization of Theorem 1.1 of [10]. In fact, here, the function $K_{1}$ can have some critical points with $(\partial K / \partial \nu)>0$.

The proof of Theorem 1.4 is based on the characterization of the critical points at infinity of the associated variational structure $J_{0}$. Assuming that $(P)$ has no solution, using [6], we know that $\Sigma^{+}$(defined in (1.2)) retracts by deformation onto set $X$ which is the union of the unstable manifolds of 
the critical points at infinity. Since $\Sigma^{+}$is a contractible set, we derive that the Euler-Poincaré characteristic of $\Sigma^{+}$is equal to 1 . Furthermore, under the assumptions $\left(H_{1}\right)-\left(H_{5}\right)$, we prove that the contribution of the critical points at infinity in the Euler-Poincare characteristic depends on the right hand side of (1.6). Hence, from their contribution formula, we obtain a contradiction with (1.6).

Remark 1.5. The assumption on the Morse index of $K_{1}$ in $\left(H_{5}\right)$ is very important. In fact, if there is one point $z \in \partial S_{+}^{4}$ with $(\partial K / \partial \nu)(z)>0$ and $\operatorname{index}\left(K_{1}, z\right) \in\{1,3\}$, then we derive that the contribution of the critical points at infinity in the Euler-Poincaré characteristic is always equal to 1. Hence, we find the same phenomenon as in the scalar curvature problem in the sphere $S^{n}, n \geq 5$ (see Bahri [2]).

Note that, if

$-\Delta K(y)-12 K(y) H(y, y)<0 \quad$ for each $y \in S_{+}^{4}$ such that $\nabla K(y)=0$,

then we have only boundary critical points at infinity. Thus under $\left(H_{1}\right),\left(H_{2}\right)$ and $\left(H_{5}\right)$, as a consequence of Theorem 1.4, we derive that $(P)$ has a solution. However, we can improve this result and, under some assumptions, we prove that the functional $J_{0}$ has to satisfy the Palais-Smale condition under a given level. In fact we have

Theorem 1.6. Let $z_{0}, y_{0}$ be such that

$$
\begin{aligned}
K_{1}\left(z_{0}\right) & =\max \left\{K_{1}(z): z \in \partial S_{+}^{4}\right\} \quad \text { and } \\
K\left(y_{0}\right) & =\max \left\{K(y): y \in S_{+}^{4}, \nabla K(y)=0\right\} .
\end{aligned}
$$

If one of the following conditions

(i) $(\partial K / \partial \nu)\left(z_{0}\right)<0, \quad$ and $\quad 2 K\left(z_{0}\right) \geq K\left(y_{0}\right)$

(ii) $-\Delta K(y)-12 K(y) H(y, y)<0, \quad$ and $\quad 2 K\left(z_{0}\right) \leq K\left(y_{0}\right)$

holds, then $(P)$ has at least one solution $w$. Furthermore, we have $J_{0}(w) \leq \overline{\mu_{0}}$ where $\overline{\mu_{0}}:=\left(S^{4}\right)^{1 / 2} \min \left\{\left(2 K\left(z_{0}\right)\right)^{-1 / 2}, K\left(y_{0}\right)^{-1 / 2}\right\}$.

We remark that if we have $(\partial K / \partial \nu)\left(z_{0}\right)<0$ and $(1.7)$ is satisfied for $y_{0}$ then one of the above conditions in Theorem 1.6 is satisfied and therefore $(P)$ has a solution. We note that, part of this result is proved in [8] (when the condition ( $i$ ) holds).

The remainder of the present paper is organized as follows. In Sect. 2, we prove Theorem 1.1. In Sect. 3, we set up the variational structure of $(P)$ and we recall some preliminaries. In Sect. 4, we perform the construction of a pseudo gradient of $J_{0}$ which satisfies some required properties. While Sect. 5 is devoted to the proofs of the other results. The proofs require some careful expansions which are given in Appendices. 


\section{Proof of Theorem 1.1}

First, let us introduce the general setting. For $\varepsilon>0$, we define the functional

$$
I_{\varepsilon}(u)=\frac{1}{2} \int_{S_{+}^{4}}|\nabla u|^{2}+\int_{S_{+}^{4}} u^{2}-\frac{1}{4-\varepsilon} \int_{S_{+}^{4}} K|u|^{4-\varepsilon}, \quad u \in H^{1}\left(S_{+}^{4}\right) .
$$

Note that if $u$ is a positive critical point of $I_{\varepsilon}$, then $u$ is a solution of $\left(P_{\varepsilon}\right)$, and inversely.

Furthermore, we mention that it will be convenient to perform some stereographic projection in order to reduce our problem to $\mathbb{R}_{+}^{4}$. Let $D^{1,2}\left(\mathbb{R}_{+}^{4}\right)$ denote the completion of $C_{c}^{\infty}\left(\overline{\mathbb{R}_{+}^{4}}\right)$ with respect to Dirichlet norm. The stereographic projection $\pi_{a}$ through a point $a \in \partial S_{+}^{4}$ induces an isometry $\iota: H^{1}\left(S_{+}^{4}\right) \rightarrow$ $D^{1,2}\left(\mathbb{R}_{+}^{4}\right)$ according to the following formula

$$
(\iota v)(x)=2\left(1+|x|^{2}\right)^{-1} v\left(\pi_{a}^{-1}(x)\right), \quad v \in H^{1}\left(S_{+}^{4}\right), x \in \mathbb{R}_{+}^{4} .
$$

In particular, one can check that the following holds true, for every $v \in H^{1}\left(S_{+}^{4}\right)$

$$
\int_{S_{+}^{4}}\left(|\nabla v|^{2}+2 v^{2}\right)=\int_{\mathbb{R}_{+}^{4}}|\nabla(\iota v)|^{2} \quad \text { and } \quad \int_{S_{+}^{4}}|v|^{4}=\int_{\mathbb{R}_{+}^{4}}|\iota v|^{4} .
$$

In the sequel, we will identify the function $K$ and its composition with the stereographic projection $\pi_{a}$. We will also identify a point $b$ of $S_{+}^{4}$ and its image by $\pi_{a}$. Moreover, it is easy to see that, using (2.1) with $\pi_{-a}$, the function $\iota \delta_{(a, \lambda)}$ is equal to

$$
\iota \delta_{(a, \lambda)}=c_{0} \frac{\lambda}{1+\lambda^{2}|x-a|^{2}} .
$$

For sake of simplicity, we will write $\delta_{(a, \lambda)}$ instead of $\iota \delta_{(a, \lambda)}$. These facts will be assumed as understood in the sequel. Let $\partial_{\nu} K$ denote the normal derivative of $K$ and let

$$
\begin{aligned}
M_{\varepsilon}= & \left\{m=\left(\alpha, \lambda, x_{1}, x_{2}, v\right) \in \mathbb{R}^{2} \times\left(\mathbb{R}_{+}^{*}\right)^{2} \times \partial S_{+}^{4} \times\left(S_{+}^{4}\right) \times H^{1}\left(S_{+}^{4}\right): v \in E_{(x, \lambda)},\right. \\
& \|v\|<\nu_{0} ;\left|\alpha_{i}^{2} K\left(x_{i}\right) \alpha_{j}^{-2} K\left(x_{j}\right)^{-1}-1\right|<\nu_{0}, \lambda_{i}>\nu_{0}^{-1}, \varepsilon \log \lambda_{i}<\nu_{0}, \forall i ; \\
& c_{0} \lambda_{2}^{2}<\lambda_{1}<c_{0}^{-1} \lambda_{2}^{2} ;\left|x_{1}-x_{2}\right|>d_{0} ; \\
& \left.\left|16 c_{3} \partial_{\nu} K\left(x_{1}\right) \lambda_{1}^{-1}-\varepsilon K\left(x_{1}\right) S_{4}\right|<\varepsilon^{1+\sigma / 2}\right\},
\end{aligned}
$$

where $\sigma, c_{0}, d_{0}$ are some suitable positive constants, $\nu_{0}$ is a small positive constant and

$$
\begin{aligned}
E_{(x, \lambda)}= & \left\{w \in H^{1}\left(S_{+}^{4}\right) /\langle w, \varphi\rangle=0\right. \\
& \left.\forall \varphi \in\left\{P \delta_{i}, \partial P \delta_{i} / \partial \lambda_{i}, \partial P \delta_{i} / \partial x_{i}^{j}, i=1,2 ; j \leq 4\right\}\right\} .
\end{aligned}
$$

Here, $x_{i}^{j}$ denotes the $j$-th component of $x_{i}$ and it is easy to get that $P \delta_{\left(x_{1}, \lambda_{1}\right)}=$ $\delta_{\left(x_{1}, \lambda_{1}\right)}$ since $x_{1} \in \partial S_{+}^{4}$. Let us define the function

$$
\Psi_{\varepsilon}: M_{\varepsilon} \rightarrow \mathbb{R} ; \quad m=(\alpha, \lambda, x, v) \mapsto I_{\varepsilon}\left(\alpha_{1} \delta_{\left(x_{1}, \lambda_{1}\right)}+\alpha_{2} P \delta_{\left(x_{2}, \lambda_{2}\right)}+v\right) .
$$

For sake of simplicity, we will write $P \delta_{i}$ instead of $P \delta_{\left(x_{i}, \lambda_{i}\right)}$. 
As said before, if $a$ belongs to $\partial S_{+}^{4}$, then we have $P \delta_{(a, \lambda)}=\delta_{(a, \lambda)}$ and therefore in our case, for $m=(\alpha, \lambda, x, v) \in M_{\varepsilon}$ we can write $u=\alpha_{1} \delta_{1}+\alpha_{2} P \delta_{2}+v$ as $u=\alpha_{1} P \delta_{1}+\alpha_{2} P \delta_{2}+v$.

As in [5], using the Euler-Lagrange's coefficients, it is easy to get the following proposition.

Proposition 2.1. Let $m=(\alpha, \lambda, x, v) \in M_{\varepsilon} \cdot m$ is a critical point of $\Psi_{\varepsilon}$ if and only if $u=\alpha_{1} \delta_{1}+\alpha_{2} P \delta_{2}+v$ is a critical point of $I_{\varepsilon}$, i.e. if and only if there exists $(A, B, C) \in \mathbb{R}^{2} \times \mathbb{R}^{2} \times\left(\mathbb{R}^{4}\right)^{2}$ such that the following holds:

$$
\begin{gathered}
\frac{\partial \Psi_{\varepsilon}}{\partial \alpha_{i}}=0, \forall i=1,2 \\
\frac{\partial \Psi_{\varepsilon}}{\partial \lambda_{i}}=B_{i}\left\langle\frac{\partial^{2} P \delta_{i}}{\partial \lambda_{i}^{2}}, v\right\rangle+\sum_{j=1}^{4} C_{i j}\left\langle\frac{\partial^{2} P \delta_{i}}{\partial x_{i}^{j} \partial \lambda_{i}}, v\right\rangle, \forall i=1,2 \quad\left(E_{\alpha_{i}}\right) \\
\frac{\partial \Psi_{\varepsilon}}{\partial x_{i}}=B_{i}\left\langle\frac{\partial^{2} P \delta_{i}}{\partial \lambda_{i} \partial x_{i}}, v\right\rangle+\sum_{j=1}^{4} C_{i j}\left\langle\frac{\partial^{2} P \delta_{i}}{\partial x_{i}^{j} \partial x_{i}}, v\right\rangle, \forall i=1,2 \\
\frac{\partial \Psi_{\varepsilon}}{\partial v}=\sum_{i=1,2}\left(A_{i} P \delta_{i}+B_{i} \frac{\partial P \delta_{i}}{\partial \lambda_{i}}+\sum_{j=1}^{4} C_{i j} \frac{\partial P \delta_{i}}{\partial x_{i}^{j}}\right) .
\end{gathered}
$$

The results of Theorem 1.1 will be obtained through a careful analysis of the previous equations on $M_{\varepsilon}$. As usual in this type of problems, we first deal with the $v$-part of $u$, in order to show that it is negligible with respect to the concentration phenomenon. The study of $\left(E_{v}\right)$ yields:

Proposition 2.2. There exists a smooth map which to any $(\varepsilon, \alpha, \lambda, x)$ such that $(\alpha, \lambda, x, 0)$ in $M_{\varepsilon}$ associates $\bar{v} \in E_{(x, \lambda)}$ such that $\|v\|<\nu_{0}$ and $\left(E_{v}\right)$ is satisfied for some $(A, B, C) \in \mathbb{R}^{2} \times \mathbb{R}^{2} \times\left(\mathbb{R}^{4}\right)^{2}$. Such a $\bar{v}$ is unique, minimizes $\Psi_{\varepsilon}(\alpha, \lambda, x, v)$ with respect to $v$ in $\left\{v \in E_{(x, \lambda)} /\|v\|<\nu_{0}\right\}$, and we have the following estimate

$$
\|\bar{v}\|=O\left(\varepsilon+\frac{1}{\lambda_{1}}+\frac{\left|\nabla K\left(x_{2}\right)\right|}{\lambda_{2}}+\frac{1}{\lambda_{2}^{2}}+\varepsilon_{12}\left(\log \varepsilon_{12}^{-1}\right)^{1 / 2}\right) .
$$

The proof becomes standard, hence we will omit it.

Proof of Theorem 1.1. The proof follows the ideas introduced in [5]. Once $\bar{v}$ is defined by Proposition 2.2, we estimate the numbers $A, B, C$ by taking the scalar product of $\left(E_{v}\right)$ with $\delta_{1}, P \delta_{2}, \partial \delta_{1} / \partial \lambda_{1}, \partial P \delta_{2} / \partial \lambda_{2}, \partial \delta_{1} / \partial x_{1}$ and $\partial P \delta_{2} / \partial x_{2}$ respectively. Thus, as in [5], we get a quasi-diagonal system in the variables $A, B$ and $C_{i}$ 's.

The other hand side is given by

$$
\frac{\partial \Psi_{\varepsilon}}{\partial \alpha_{i}}=\left\langle\frac{\partial \Psi_{\varepsilon}}{\partial v}, P \delta_{i}\right\rangle ; \quad \frac{1}{\alpha_{i}} \frac{\partial \Psi_{\varepsilon}}{\partial \lambda_{i}}=\left\langle\frac{\partial \Psi_{\varepsilon}}{\partial v}, \frac{\partial P \delta_{i}}{\partial \lambda_{i}}\right\rangle ; \quad \frac{1}{\alpha_{i}} \frac{\partial \Psi_{\varepsilon}}{\partial x_{i}}=\left\langle\frac{\partial \Psi_{\varepsilon}}{\partial v}, \frac{\partial P \delta_{i}}{\partial x_{i}}\right\rangle .
$$


Using Propositions 6.2 and 6.3, some computations yield

$$
\frac{\partial \Psi_{\varepsilon}}{\partial \alpha_{1}}=-S_{4} \beta_{1}+V_{\alpha_{1}}(\varepsilon, \alpha, \lambda, x), \quad \frac{\partial \Psi_{\varepsilon}}{\partial \alpha_{2}}=-2 S_{4} \beta_{2}+V_{\alpha_{2}}(\varepsilon, \alpha, \lambda, x),
$$

with $\beta_{1}=\alpha_{1}-1 / K(z)^{\frac{1}{2}}, \beta_{2}=\alpha_{2}-1 / K(y)^{\frac{1}{2}}$ and $V_{\alpha_{i}}$ is a smooth function which satisfies

$$
\begin{aligned}
V_{\alpha_{i}}= & O\left(\beta_{i}^{2}+\varepsilon \log \lambda_{i}+(\text { if } i=1)\left(\lambda_{1}^{-1}+\left|x_{1}-z\right|^{2}\right)\right. \\
& \left.+(\text { if } i=2)\left(\lambda_{2}^{-2}+\left|x_{2}-y\right|^{2}\right)\right) .
\end{aligned}
$$

In the same way we get

$$
\begin{aligned}
\frac{\partial \Psi_{\varepsilon}}{\partial \lambda_{1}}= & \frac{1}{K(z)}\left(\frac{2 c_{3}}{\lambda_{1}^{2}} \frac{\partial K}{\partial \nu}\left(x_{1}\right)+\frac{\varepsilon K\left(x_{1}\right) S_{4}}{8 \lambda_{1}}\right)+V_{\lambda_{1}}(\varepsilon, \alpha, \lambda, x), \\
\frac{\partial \Psi_{\varepsilon}}{\partial \lambda_{2}}= & \frac{1}{K(y)}\left(\frac{\varepsilon K\left(x_{2}\right) S_{4}}{4 \lambda_{2}}+\frac{c_{2}}{4}\left(\frac{\Delta K\left(x_{2}\right)}{3 K\left(x_{2}\right)}+4 H\left(x_{2}, x_{2}\right)\right) \frac{1}{\lambda_{2}^{3}}\right) \\
& +V_{\lambda_{2}}(\varepsilon, \alpha, \lambda, x), \\
\frac{\partial \Psi_{\varepsilon}}{\partial x_{1}}= & -2 c_{5} \nabla_{T} K\left(x_{1}\right)+V_{x_{1}}(\varepsilon, \alpha, \lambda, x) \text { and } \\
\frac{\partial \Psi_{\varepsilon}}{\partial x_{2}}= & -c_{8} \nabla K\left(x_{2}\right)+V_{x_{2}}(\varepsilon, \alpha, \lambda, x),
\end{aligned}
$$

where the constants $c_{i}$ are defined in the appendices, $V_{\lambda_{i}}$ and $V_{x_{i}}$ are smooth functions satisfying

$$
\begin{aligned}
V_{\lambda_{i}}= & O\left(\lambda_{i}^{-1}\left(\lambda_{i}^{-i-1}+\left|x_{2}-y\right|^{2} \lambda_{2}^{-2}+\varepsilon^{2} \log \lambda_{i}+\varepsilon \lambda_{i}^{-i} \log \lambda_{i}\right)\right. \\
& \left.+\left(|\beta|+\varepsilon+\left|x_{i}-\bar{a}_{i}\right|^{2}\right)\left(\varepsilon \lambda_{i}^{-1}+\lambda_{i}^{-i-1}\right)\right), \\
V_{x_{i}}= & O\left(\lambda_{i}^{-1}+\left(|\beta|+\varepsilon \log \lambda_{i}+\left|x_{i}-\bar{a}_{i}\right|^{2}\right)\left|x_{i}-\bar{a}_{i}\right|\right), \\
& \text { where } \bar{a}_{1}=z \text { and } \bar{a}_{2}=y .
\end{aligned}
$$

Using the previous estimates, the solution of the system in $A, B$ and $C$ shows that

$$
\left\{\begin{array}{l}
A_{i}=O\left(|\beta|+\varepsilon \log \lambda_{i}+(\text { if } i=1)\left(\frac{1}{\lambda_{1}}+\left|x_{1}-z\right|^{2}\right)+(\text { if } i=2)\left(\frac{1}{\lambda_{2}^{2}}+\left|x_{2}-y\right|^{2}\right)\right), \\
B_{1}=O\left(\varepsilon^{1+\sigma / 2} \lambda_{1}\right) ; \quad B_{2}=O\left(\frac{1}{\lambda_{2}}+\varepsilon \lambda_{2}\right) \\
C_{1}=O\left(\frac{\left|x_{1}-z\right|}{\lambda_{1}^{2}}+\frac{1}{\lambda_{1}^{3}}\right) ; \quad C_{2}=O\left(\frac{\left|x_{2}-y\right|}{\lambda_{2}^{2}}+\frac{1}{\lambda_{2}^{3}}\right) .
\end{array}\right.
$$

This allows us to evaluate the right hand side in the equations $\left(E_{\lambda_{i}}\right)$ and $\left(E_{x_{i}}\right)$. Now, we consider a point $(z, y) \in \partial S_{+}^{4} \times S_{+}^{4}$ such that $z$ and $y$ are nondegenerate critical points of $K_{1}$ and $K$ respectively. We set

$$
\begin{array}{r}
\frac{1}{\lambda_{1}}=\frac{\varepsilon S_{4}}{16 c_{3}} K(z)\left(\frac{\partial K}{\partial \nu}(z)\right)^{-1}\left(1+\zeta_{1}\right) ; \quad x_{1}=z+\xi_{1} ; \\
\frac{1}{\lambda_{2}}=\varepsilon^{\frac{1}{2}} \Lambda\left(1+\zeta_{2}\right) ; \quad x_{2}=y+\xi_{2},
\end{array}
$$

where $\Lambda$ verifies : $c_{2} \Lambda^{2}\left(\frac{\Delta K(y)}{3 K(y)}+4 H(y, y)\right)+S_{4} K(y)=0, \zeta_{i} \in \mathbb{R}$ and $\left(\xi_{1}, \xi_{2}\right) \in$ $\mathbb{R}^{3} \times \mathbb{R}^{4}$ are assumed to be small. With these changes of variables and using 
$(2.3),\left(E_{\alpha_{i}}\right)$ is equivalent to

$$
\beta_{i}=V_{\alpha_{i}}(\varepsilon, \beta, \zeta, \xi)=O\left(\beta^{2}+\varepsilon|\log \varepsilon|+|\xi|^{2}\right) .
$$

Now, using (2.4), an easy computation shows that

$$
\begin{aligned}
\frac{2 c_{3}}{\lambda_{1}^{2}} & \frac{\partial K}{\partial \nu}\left(z+\xi_{1}\right)+\frac{\varepsilon K\left(z+\xi_{1}\right) S_{4}}{8 \lambda_{1}} \\
= & \frac{\varepsilon^{2} S_{4}^{2} K(z)^{2}}{128 c_{3}}\left(-\left(\frac{\partial K}{\partial \nu}(z)\right)^{-1} \zeta_{1}+\left(\frac{\partial K}{\partial \nu}(z)\right)^{-2} D^{2} K_{1}(z)\left(e_{4}, \xi_{1}\right)\right) \\
& +O\left(\varepsilon^{2}\left(\zeta_{1}^{2}+\left|\xi_{1}\right|^{2}\right)\right) .
\end{aligned}
$$

This implies that $\left(E_{\lambda_{1}}\right)$ is equivalent, while using (2.7) and (2.9), to

$$
\begin{aligned}
& -\zeta_{1}+\left(\frac{\partial K}{\partial \nu}(z)\right)^{-1} D^{2} K_{1}(z)\left(e_{4}, \xi_{1}\right)=V_{\lambda_{1}}(\varepsilon, \beta, \zeta, \xi) \\
& =O\left(\varepsilon|\log \varepsilon|+|\beta|^{2}+\zeta_{1}^{2}+|\xi|^{2}\right) .
\end{aligned}
$$

Using (2.9), an easy computation shows that

$$
\begin{aligned}
& \frac{\varepsilon K\left(y+\xi_{2}\right) S_{4}}{4 \lambda_{2}}+\frac{c_{2}}{4}\left(\frac{\Delta K\left(y+\xi_{2}\right)}{3 K\left(y+\xi_{2}\right)}+4 H\left(y+\xi_{2}, y+\xi_{2}\right)\right) \frac{1}{\lambda_{2}^{3}} \\
& =\frac{\varepsilon^{3 / 2} \Lambda^{3} c_{2}}{2}\left(\frac{\Delta K(y)}{3 K(y)}+4 H(y, y)\right) \zeta_{2} \\
& \quad+\frac{\varepsilon^{3 / 2} \Lambda^{3} c_{2}}{4}\left(\frac{1}{3 K(y)} \nabla(\Delta K)(y)+8 \frac{\partial H}{\partial x_{2}}(y, y)\right) \xi_{2} \\
& \quad+O\left(\varepsilon^{3 / 2}\left(\zeta_{2}^{2}+\left|\xi_{2}\right|^{2}\right)\right) .
\end{aligned}
$$

This implies that $\left(E_{\lambda_{2}}\right)$ is equivalent, while using (2.7) and (2.9), to

$$
\begin{aligned}
& \left(\frac{\Delta K(y)}{3 K(y)}+4 H(y, y)\right) \zeta_{2}+\frac{1}{2}\left(\frac{1}{3 K(y)} \nabla(\Delta K)(y)+8 \frac{\partial H}{\partial x_{2}}(y, y)\right) \xi_{2} \\
& \quad=V_{\lambda_{2}}(\varepsilon, \beta, \zeta, \xi)=O\left(|\beta|^{2}+\zeta_{2}^{2}+|\xi|^{2}+\varepsilon^{1 / 2}\right) .
\end{aligned}
$$

Using (2.6), (2.8) and (2.9), $\left(E_{x_{1}}\right)$ is equivalent to

$$
D^{2} K_{1}(z) \xi_{1}=V_{x_{1}}(\varepsilon, \beta, \zeta, \xi)=O\left(\varepsilon^{1 / 2}+|\beta|^{2}+|\zeta|^{2}+|\xi|^{2}\right)
$$

Lastly, using (2.6), (2.8) and (2.9), $\left(E_{x_{2}}\right)$ is equivalent to

$$
D^{2} K(y) \xi_{2}=V_{x_{2}}(\varepsilon, \beta, \zeta, \xi)=O\left(\varepsilon^{1 / 2}+|\beta|^{2}+|\zeta|^{2}+|\xi|^{2}\right) .
$$

This system may also be written as

$$
\beta=V(\varepsilon, \beta, \zeta, \xi), \quad \text { and } \quad L(\zeta, \xi)=W(\varepsilon, \beta, \zeta, \xi),
$$

where $L$ is a fixed linear operator on $\mathbb{R}^{9}$ defined by the left hand sides of (2.10)-(2.13), and $V, W$ are smooth functions satisfying

$$
\left\{\begin{array}{l}
V(\varepsilon, \beta, \zeta, \xi)=O\left(\varepsilon^{1 / 2}+|\beta|^{2}+|\xi|^{2}\right), \\
W(\varepsilon, \beta, \zeta, \xi)=O\left(\varepsilon^{1 / 2}+|\beta|^{2}+|\zeta|^{2}+|\xi|^{2}\right) .
\end{array}\right.
$$


Moreover, a simple computation shows that the determinant of $L$ is not equal to zero. Hence $L$ is invertible, and Brouwer's fixed point theorem shows that (2.14) has a solution $\left(\beta^{\varepsilon}, \zeta^{\varepsilon}, \xi^{\varepsilon}\right)$ for $\varepsilon$ small enough, such that

$$
\left|\beta^{\varepsilon}\right|=O\left(\varepsilon^{1 / 2}\right) ; \quad\left|\zeta^{\varepsilon}\right|=O\left(\varepsilon^{1 / 2}\right) ; \quad\left|\xi^{\varepsilon}\right|=O\left(\varepsilon^{1 / 2}\right) .
$$

Hence, we have constructed $m^{\varepsilon}=\left(\alpha_{1}^{\varepsilon}, \alpha_{2}^{\varepsilon}, \lambda_{1}^{\varepsilon}, \lambda_{2}^{\varepsilon}, x_{1}^{\varepsilon}, x_{2}^{\varepsilon}\right)$ such that $u_{\varepsilon}:=$ $\sum \alpha_{i}^{\varepsilon} P \delta_{\left(x_{i}^{\varepsilon}, \lambda_{i}^{\varepsilon}\right)}+\overline{v_{\varepsilon}}$, is a critical point of $I_{\varepsilon}$ (by Proposition 2.1), i.e $u_{\varepsilon}$ satisfies

$$
-\Delta u_{\varepsilon}+2 u_{\varepsilon}=K\left|u_{\varepsilon}\right|^{2-\varepsilon} u_{\varepsilon} \quad \text { in } S_{+}^{4}, \quad \partial u_{\varepsilon} / \partial \nu=0 \text { on } \partial S_{+}^{4} .
$$

Since $\left\|u_{\varepsilon}^{-}\right\|$is very small (where $u_{\varepsilon}^{-}=\max \left(0,-u_{\varepsilon}\right)$ ), multiplying the Eq. (2.15) by $u_{\varepsilon}^{-}$and integrating on $S_{+}^{4}$ we derive that $u_{\varepsilon}^{-}=0$ and by the maximum principle we obtain: $u_{\varepsilon}>0$.

\section{Preliminary results}

We define the functional

$$
I_{0}(u)=\frac{1}{2} \int_{S_{+}^{4}}|\nabla u|^{2}+\int_{S_{+}^{4}} u^{2}-\frac{1}{4} \int_{S_{+}^{4}} K|u|^{4}, \quad u \in H^{1}\left(S_{+}^{4}\right) .
$$

Note that if $u$ is a positive critical point of $I_{0}$, then $u$ is a solution of $(P)$ and inversely.

For $u \in \Sigma^{+}$, in a first step, we can minimize, on the radial direction and it is easy to prove that, for each $u \in \Sigma^{+}$, there exists only one critical point which will be denoted by $\pi(u)$. Hence the study will be reduced in the sphere $\Sigma^{+}$of the functional $I_{0}(\pi(u) u$ ) which has the same critical points as the functional $J_{0}$ associated to $(P)$ defined by $(1.2)$.

The Palais-Smale condition fails to be satisfied for $J_{0}$ on $\Sigma^{+}$. Its failure has been studied by various authors (see Brezis-Coron [12], Lions [22], Struwe [24]) and it becomes well known that: assuming that $J_{0}$ has no critical points in $\Sigma^{+}$, then it satisfies the Palais-Smale condition outside $\cup_{p} V(p, \varepsilon)$, where for $\varepsilon>0$ small enough and $p \in \mathbb{N}^{*}$

$$
\begin{aligned}
V(p, \varepsilon):=\left\{\begin{array}{l}
u \in \Sigma^{+} / \exists a_{i} \in \overline{S_{+}^{4}}, \exists \lambda_{i}>\varepsilon^{-1}, \exists \alpha_{i}>0, \text { for } i=1, \ldots, p \text { such that } \\
\\
\left\|u-\sum_{i=1}^{p} \alpha_{i} \delta_{i}\right\|<\varepsilon,\left|\frac{\alpha_{i}^{2} K\left(a_{i}\right)}{\alpha_{j}^{2} K\left(a_{j}\right)}-1\right|<\varepsilon, \varepsilon_{i j}<\varepsilon \text { and } \lambda_{i} d_{i}<\varepsilon \text { or } \\
\lambda_{i} d_{i}>\varepsilon^{-1}
\end{array}\right\},
\end{aligned}
$$

where $\delta_{i}=\delta_{\left(a_{i}, \lambda_{i}\right)}, d_{i}=d\left(a_{i}, \partial S_{+}^{4}\right)$ and $\varepsilon_{i j}^{-1}=\lambda_{i} / \lambda_{j}+\lambda_{j} / \lambda_{i}+\lambda_{i} \lambda_{j}(1-$ $\left.\cos d\left(a_{i}, a_{j}\right)\right) / 2$. 
Remark 3.1. Note that the corresponding $\varepsilon_{i j}$ when we use the variables $a_{i}$ in $\mathbb{R}_{+}^{4}$ is defined by

$$
\varepsilon_{i j}=\left(\frac{\lambda_{i}}{\lambda_{j}}+\frac{\lambda_{j}}{\lambda_{i}}+\lambda_{i} \lambda_{j}\left|a_{i}-a_{j}\right|^{2}\right)^{-1} .
$$

Furthermore, the set $V(p, \varepsilon)$ is parametrized as: For each $u \in V(p, \varepsilon)$, there exist a unique $\left(\alpha_{1}, \ldots, \alpha_{p}, a_{1}, \ldots, a_{p}, \lambda_{1}, \ldots, \lambda_{p}\right)$ (up to permutation) and $v \in H^{1}\left(S_{+}^{4}\right)$ satisfying

$$
\|v\| \leq \varepsilon,\langle v, \psi\rangle=0 \text { for } \psi \in\left\{\delta_{i}, \frac{\partial \delta_{i}}{\partial \lambda_{i}}, \frac{\partial \delta_{i}}{\partial a_{i}}, P \delta_{j}, \frac{\partial P \delta_{j}}{\partial \lambda_{j}}, \frac{\partial P \delta_{j}}{\partial a_{j}} \quad: i \leq q, j>q\right\},
$$

such that

$$
u=\sum_{i=1}^{q} \alpha_{i} \delta_{\left(a_{i}, \lambda_{i}\right)}+\sum_{i=q+1}^{p} \alpha_{i} P \delta_{\left(a_{i}, \lambda_{i}\right)}+v .
$$

We also have the following proposition whose proof is similar, up to modifications, to the corresponding statements in [2].

Proposition 3.2. There exists a $C^{1}$ map which, to each $\left(\alpha_{1}, \ldots, \alpha_{p}, a_{1}, \ldots, a_{p}\right.$, $\left.\lambda_{1}, \ldots, \lambda_{p}\right)$ such that $w:=\sum_{i=1}^{q} \alpha_{i} \delta_{\left(a_{i}, \lambda_{i}\right)}+\sum_{i=q+1}^{p} \alpha_{i} P \delta_{\left(a_{i}, \lambda_{i}\right)} \in V(p, \varepsilon)$ associates $\bar{v}=\bar{v}_{\left(\alpha_{i}, a_{i}, \lambda_{i}\right)}$ satisfying

$$
J_{0}(w+\bar{v})=\min \left\{J_{0}(w+v), v \text { satisfies }\left(V_{0}\right)\right\} .
$$

Moreover, there exists a constant $c>0$ such that the following holds

$$
\|\bar{v}\| \leq c\left(\sum_{i \leq q} \frac{1}{\lambda_{i}}+\sum_{i>q} \frac{\left|\nabla K\left(a_{i}\right)\right|}{\lambda_{i}}+\sum_{i>q} \frac{1}{\left(\lambda_{i} d_{i}\right)^{2}}+\sum_{k \neq r} \varepsilon_{k r} \log \left(\varepsilon_{k r}^{-1}\right)^{1 / 2}\right) .
$$

\section{Construction of a pseudo gradient flow in $V(p, \varepsilon)$}

In this section we are going to construct a global pseudo gradient flow for the functional $J_{0}$ under assumptions $\left(H_{1}-H_{4}\right)$ on $K$. Along its flow lines there can be only finitely many isolated blow up points. Such a flow is defined by combining two basic facts. On the one hand, the first one comes from the Morse Lemma at infinity which moves points and concentrations as follows: points move according to $\nabla K$ if they are interior points and along $\nabla K_{1}$ if they are boundary points, concentrations move so as to decrease the functional $J_{0}$. On the other hand, there is another pseudo gradient when the points are very close and the total interaction $\sum \varepsilon_{i j}$ is large with respect to $\sum \frac{1}{\lambda_{i}}$. We need to convex-combine both flows. The obtained pseudo gradient has the following property: when the flow lines, starting from $V(p, \varepsilon / 2)$, leave $V(p, \varepsilon)$ the functional $J_{0}$ will decrease by a fixed constant which depends only on $\varepsilon$. Hence the kind of travel cannot exist infinite times.

Following A. Bahri we introduce the following definitions and notations 
Definition 4.1. A critical point at infinity of $J_{0}$ in $V(p, \varepsilon)$ is a limit of a flow line $u(s)$ of

$$
\frac{\partial u}{\partial s}=-\nabla J_{0}(u) \quad \text { and } \quad u(0)=u_{0} \in \Sigma^{+}
$$

such that $u(s)$ remains in $V(p, \varepsilon(s))$ for $s \geq s_{0}$, where $\varepsilon(s)$ is some function tending to zero when $s \rightarrow \infty$. Using Remark 3.1, $u(s)$ can be written as:

$$
u(s)=\sum_{i \leq p} \alpha_{i}(s) P \delta_{\left(a_{i}(s), \lambda_{i}(s)\right)}+v(s) .
$$

Denoting $a_{i}:=\lim _{s \rightarrow \infty} a_{i}(s)$ and $\alpha_{i}:=\lim _{s \rightarrow \infty} \alpha_{i}(s)$, following the notation of A. Bahri, we denote such a critical point at infinity by

$$
\left(a_{1}, \ldots, a_{p}\right)_{\infty} \text { or } \sum_{i \leq p} \alpha_{i} P \delta_{\left(a_{i}, \infty\right)} .
$$

For such a critical point at infinity once a Morse type reduction is performed, are associated stable and unstable manifolds see $[2,3]$.

As a by product of the construction of our pseudo gradient, we are able to perform a Morse Lemma at infinity and therefore, we identify the critical points at infinity of our problem.

Proposition 4.2. For $p \geq 1$, there exists a pseudo gradient $W$ so that the following holds : There is a constant $c>0$ independent of $u=\sum_{i=1}^{q} \alpha_{i} \delta_{i}+$ $\sum_{j=q+1}^{p} \alpha_{j} P \delta_{j} \in V(p, \varepsilon)$ so that

(i) $\left\langle-\nabla J_{0}(u), W\right\rangle$

$$
\begin{aligned}
& \geq c \sum_{i \leq q}\left(\sum_{j \neq i} \varepsilon_{i j}^{3 / 2}+\frac{1}{\lambda_{i}^{3 / 2}}+\left|1-J_{0}(u)^{2} \alpha_{i}^{2} K\left(a_{i}\right)\right|^{\frac{3}{2}}\right) \\
& +c \sum_{i>q}\left(\sum_{j \neq i} \varepsilon_{i j}+\frac{1}{\left(\lambda_{i} d_{i}\right)^{2}}+\frac{\left|\nabla K\left(a_{i}\right)\right|}{\lambda_{i}}\right)
\end{aligned}
$$

(ii) $\left\langle-\nabla J_{0}(u+\bar{v}), W+\frac{\partial \bar{v}}{\partial\left(\alpha_{i}, a_{i}, \lambda_{i}\right)}(W)\right\rangle$

$$
\begin{aligned}
& \geq c \sum_{i \leq q}\left(\sum_{j \neq i} \varepsilon_{i j}^{3 / 2}+\frac{1}{\lambda_{i}^{3 / 2}}+\left|1-J_{0}(u)^{2} \alpha_{i}^{2} K\left(a_{i}\right)\right|^{\frac{3}{2}}\right) \\
& \quad+c \sum_{i>q}\left(\sum_{j \neq i} \varepsilon_{i j}+\frac{1}{\left(\lambda_{i} d_{i}\right)^{2}}+\frac{\left|\nabla K\left(a_{i}\right)\right|}{\lambda_{i}}\right)
\end{aligned}
$$

(iii) $|W|$ is bounded. Furthermore, the only case where the maximum of the $\lambda_{i}$ 's is not bounded is when each point $a_{i}$ of the boundary is close to a critical point $z_{j_{i}}$ of $K_{1}$ with $(\partial K / \partial \nu)\left(z_{j_{i}}\right)>0$ and $z_{j_{i}} \neq z_{j_{k}}$ for $i \neq k$, and each point $a_{i}$ in $S_{+}^{4}$ is close to a critical point $y_{j_{i}}$ of $K$ with $j_{i} \neq j_{k}$ for $i \neq k$ and $\rho\left(y_{j_{1}}, \ldots, y_{j_{s}}\right)>0$, where $s=p-q$ and $\rho\left(y_{j_{1}}, \ldots, y_{j_{s}}\right)$ denotes the least eigenvalue of $M\left(y_{j_{1}}, \ldots, y_{j_{s}}\right)$.

Before proving Proposition 4.2, we prove Theorem 1.2 which gives a construction of a pseudo gradient when the concentration points are close to the same critical point of $K_{1}$ with $\partial K / \partial \nu>0$. 
Proof of Theorem 1.2. The required pseudo gradient will be defined by using the variables $\alpha_{i}, a_{i}$ and $\lambda_{i}$. Concerning the $\alpha_{i}$ 's variables, we define the vector field

$$
Z_{i}^{\alpha}=\psi\left(\lambda_{i}\left(1-J_{0}(u)^{2} \alpha_{i}^{2} K\left(a_{i}\right)\right)\right) \delta_{i}
$$

where $\psi$ is a $C^{\infty}$ function which satisfies

$$
\begin{aligned}
& t \psi(t) \leq 0, \quad \psi(t)=-1 \quad \text { if } t>2, \quad \psi(t)=0 \quad \text { if }|t| \leq 1 \quad \text { and } \psi(t)=1 \\
& \quad \text { if } t<-2 .
\end{aligned}
$$

Using the Appendix 2 [Proposition 7.2], we derive

$$
\begin{aligned}
\left\langle-\nabla J_{0}(u), Z_{i}^{\alpha}\right\rangle \geq c\left|\psi\left(\lambda_{i}\left(1-J_{0}(u)^{2} \alpha_{i}^{2} K\left(a_{i}\right)\right)\right)\right| & {\left[\left|1-J_{0}(u)^{2} \alpha_{i}^{2} K\left(a_{i}\right)\right|+O\left(\frac{1}{\lambda_{i}}+\sum_{j \neq i} \varepsilon_{i j}\right)\right] . }
\end{aligned}
$$

Without loss of generality, we can assume that $\lambda_{1} \leq \cdots \leq \lambda_{q}$. Let

$$
I:=\{1\} \cup\left\{i \leq q: \quad \lambda_{k} \leq M \lambda_{k-1}, \forall k \leq i\right\},
$$

where $M$ is a large positive constant.

The set $I$ corresponds to the indices $i$ so that $\lambda_{i} / \lambda_{1}$ is bounded. Four cases may occur

Case 1. $I=\{1\}$. We define the following vector fields

$$
Y_{11}=\lambda_{1} \frac{\partial \delta_{1}}{\partial \lambda_{1}}, \quad Y_{12}=-\sum_{i>1} \lambda_{i} \frac{\partial \delta_{i}}{\partial \lambda_{i}} 2^{i} \quad \text { and } \quad Y_{13}=\sum_{i \leq q} Z_{i}^{\alpha} .
$$

We remark that for each $i \neq j$ with $\lambda_{i} \leq c \lambda_{j}$ we have

$$
-\lambda_{j} \frac{\partial \varepsilon_{i j}}{\partial \lambda_{j}}=\varepsilon_{i j}\left(1-2 \frac{\lambda_{i}}{\lambda_{j}} \varepsilon_{i j}\right) \geq \varepsilon_{i j}(1+o(1)) .
$$

Moreover, the above formula is also true if we have $\lambda_{l}\left|a_{i}-a_{j}\right| \rightarrow+\infty$ for $l=i, j$ (the above assumption implies that $\varepsilon_{i j} \lambda_{i} / \lambda_{j}$ is small). However, in the general case, we cannot control the sign of $-\lambda_{j} \partial \varepsilon_{i j} / \partial \lambda_{j}$. But, an easy computation implies that $\left|\lambda_{i} \partial \varepsilon_{i j} / \partial \lambda_{i}\right| \leq c \varepsilon_{i j}$ and

$$
-\lambda_{i} \frac{\partial \varepsilon_{i j}}{\partial \lambda_{i}}-2 \lambda_{j} \frac{\partial \varepsilon_{i j}}{\partial \lambda_{j}} \geq \varepsilon_{i j}(1+o(1)) \text { for } \lambda_{i} \leq \lambda_{j} .
$$

Thus, using the Appendix 2 [Proposition 7.2], we get

$$
\begin{aligned}
& \left\langle-\nabla J_{0}(u), Y_{11}\right\rangle \geq \frac{c}{\lambda_{1}}+O\left(\sum_{j \neq 1} \varepsilon_{1 j}+\sum_{k \neq r} \varepsilon_{k r}^{2}\right), \\
& \left\langle-\nabla J_{0}(u), Y_{12}\right\rangle \geq c \sum_{i>1}\left(\sum_{j \neq i} \varepsilon_{i j}+O\left(\frac{1}{\lambda_{i}}\right)\right)+O\left(\sum_{k \neq r} \varepsilon_{k r}^{2}\right) .
\end{aligned}
$$

Observe that, if for some indices $i$ we have $\left|\psi\left(\lambda_{i}\left(1-J_{0}(u)^{2} \alpha_{i}^{2} K\left(a_{i}\right)\right)\right)\right|<1$, then we get $\lambda_{i}\left|1-J_{0}(u)^{2} \alpha_{i}^{2} K\left(a_{i}\right)\right| \leq 2$ and therefore we can make appear $\left|1-J_{0}(u)^{2} \alpha_{i}^{2} K\left(a_{i}\right)\right|$ from $1 / \lambda_{1}$ which exists in the lower bound of (4.7). 
In the other case, we have $\left|\psi\left(\lambda_{i}\left(1-J_{0}(u)^{2} \alpha_{i}^{2} K\left(a_{i}\right)\right)\right)\right| \geq 1$ and therefore $\left|1-J_{0}(u)^{2} \alpha_{i}^{2} K\left(a_{i}\right)\right|$ appears in (4.4).

Our global vector field in this case is $Y_{k}^{1}=\sqrt{M} Y_{11}+M Y_{12}+Y_{13}$. Thus, we derive

$$
\left\langle-\nabla J_{0}(u), Y_{k}^{1}\right\rangle \geq c \sum_{i \leq q}\left(\sum_{j \neq i} \varepsilon_{i j}+\frac{1}{\lambda_{i}}+\left|1-J_{0}(u)^{2} \alpha_{i}^{2} K\left(a_{i}\right)\right|\right) .
$$

We remark that $\lambda_{q}$ which is the maximum of the $\lambda_{i}$ 's is a decreasing function along the flow lines generated by $Y_{k}^{1}$.

Case 2. $I$ contains at least two indices and there exists $j_{0} \in I$ satisfying

$$
c_{2} \sum_{i \in I, i \neq j_{0}} \varepsilon_{i j_{0}} \geq \frac{1}{(1-\varrho / 2)} \frac{4 c_{3}}{K\left(z_{k}\right)} \frac{1}{\lambda_{j_{0}}} \frac{\partial K}{\partial \nu}\left(z_{k}\right)
$$

where $\varrho$ is a positive constant which will be defined below (see (4.31)) and the constants $c_{i}$ are defined in Proposition 6.2.

Let $F:=\{i \in I: i$ satisfies (4.10) $\}$. We define the following vector fields

$$
\begin{aligned}
Y_{21} & =-\sum_{i \in F} \lambda_{i} \frac{\partial \delta_{i}}{\partial \lambda_{i}}, \quad Y_{22}=-m \sum_{i \in I \backslash F} \lambda_{i} \frac{\partial \delta_{i}}{\partial \lambda_{i}}-\sqrt{M} \sum_{i \notin I} \lambda_{i} \frac{\partial \delta_{i}}{\partial \lambda_{i}} 2^{i} \text { and } \\
Y_{23} & =\sum_{i \leq q} Z_{i}^{\alpha},
\end{aligned}
$$

where $m$ is a small positive constant such that $m=o\left(\varrho / M^{q}\right)$ and $\varrho M$ is large. Using the Appendix 2 [Proposition 7.2], we get

$$
\begin{aligned}
\left\langle-\nabla J_{0}(u), Y_{21}\right\rangle= & J_{0}(u) \sum_{i \in F}\left(-c_{2} \sum_{j \leq q, j \neq i} \alpha_{j} \lambda_{i} \frac{\partial \varepsilon_{i j}}{\partial \lambda_{i}}-4 c_{3} J_{0}(u)^{2} \frac{\alpha_{i}^{3}}{\lambda_{i}} \frac{\partial K}{\partial \nu}\left(a_{i}\right)\right) \\
& +R,
\end{aligned}
$$

where $R=O\left(\sum_{k \neq r} \varepsilon_{k r}^{2}+\sum_{i \leq q} \frac{1}{\lambda_{i}^{2}}\right)$.

Observe that for each $i \leq q$, we have $K\left(a_{i}\right)=K\left(z_{k}\right)+o(1), \alpha_{i}=\alpha_{j}+o(1)$, $J_{0}(u)^{2} \alpha_{i}^{2} K\left(a_{i}\right)=1+o(1)$ and $(\partial K / \partial \nu)\left(a_{i}\right)=(\partial K / \partial \nu)\left(z_{k}\right)+o(1)>c>0$ if we choose $\mu$ small enough. Using (4.5) for $i, j \in I$, thus, (4.11) becomes

$$
\begin{aligned}
\left\langle-\nabla J_{0}(u), Y_{21}\right\rangle= & \sum_{i \in F}\left(\frac{c_{2}(1+o(1))}{K\left(z_{k}\right)^{1 / 2}} \sum_{j \in I} \varepsilon_{i j}-\frac{4 c_{3}(1+o(1))}{\lambda_{i} K\left(z_{k}\right)^{3 / 2}} \frac{\partial K}{\partial \nu}\left(z_{k}\right)\right. \\
& \left.+O\left(\sum_{j \notin I} \varepsilon_{i j}\right)\right)+R .
\end{aligned}
$$

From (4.10), we derive

$$
\left\langle-\nabla J_{0}(u), Y_{21}\right\rangle \geq \sum_{i \in F}\left(c \sum_{j \in I} \varepsilon_{i j}+\frac{c}{\lambda_{i}}+O\left(\sum_{j \notin I} \varepsilon_{i j}\right)\right)+O\left(\sum_{k \neq r} \varepsilon_{k r}^{2}\right) .
$$


For the vector $Y_{22}$, using (4.6) and the Appendix 2 [Proposition 7.2], we get

$$
\begin{aligned}
\left\langle-\nabla J_{0}(u), Y_{22}\right\rangle \geq & m \sum_{i \in I \backslash F}\left(\sum_{j \neq i} \varepsilon_{i j}+O\left(\frac{1}{\lambda_{i}}\right)\right)+\sqrt{M} \sum_{i \notin I}\left(\sum_{j \neq i} \varepsilon_{i j}+O\left(\frac{1}{\lambda_{i}}\right)\right) \\
& +O\left(\sum_{k \neq r} \varepsilon_{k r}^{2}\right) .
\end{aligned}
$$

Let $Y_{k}^{2}=M^{\prime}\left(Y_{21}+Y_{22}\right)+Y_{23}$, where $M^{\prime}$ is a large positive constant. Note that all the $\lambda_{i}$ 's variables are decreasing functions along the flow lines. Using (4.12), (4.13) and (4.4) as in the proof of (4.9), it is easy to show that

$$
\left\langle-\nabla J_{0}(u), Y_{k}^{2}\right\rangle \geq c \sum_{i \leq q}\left(\sum_{j \neq i} \varepsilon_{i j}+\frac{1}{\lambda_{i}}+\left|1-J_{0}(u)^{2} \alpha_{i}^{2} K\left(a_{i}\right)\right|\right) .
$$

Case 3. $I$ contains at least two indices, $z_{k}$ is a local maximum point of $K_{1}$ and we have

$$
c_{2} \sum_{i \in I, i \neq j} \varepsilon_{i j}<\frac{1}{(1-\varrho)} \frac{4 c_{3}}{K\left(z_{k}\right)} \frac{1}{\lambda_{j}} \frac{\partial K}{\partial \nu}\left(z_{k}\right) \quad \text { for all } j \in I .
$$

In this case, we need to introduce the sets

$$
\begin{aligned}
& \Gamma_{1}=\left\{j \notin I \text { s.t. } c_{2} \sum_{i \neq j} \varepsilon_{i j} \geq \frac{1}{(1-\varrho / 2)} \frac{4 c_{3}}{K\left(z_{k}\right)} \frac{1}{\lambda_{j}} \frac{\partial K}{\partial \nu}\left(z_{k}\right)\right\} \quad \text { and } \\
& \Gamma_{2}=\left\{j \leq q: j \notin I \cup \Gamma_{1}\right\} .
\end{aligned}
$$

We define the vector field

$$
Y_{31}=\sum_{i \in I} \frac{1}{\lambda_{h}} \frac{\partial \delta_{i}}{\partial a_{i}} \frac{\nabla_{T} K\left(a_{i}\right)}{\sum_{l \in I}\left|\nabla_{T} K\left(a_{l}\right)\right|},
$$

where $h \in I$ such that $\left|\nabla_{T} K\left(a_{h}\right)\right|=\max \left\{\left|\nabla_{T} K\left(a_{i}\right)\right|, i \in I\right\}$.

From the Appendix 2 [Proposition 7.2], we get

$$
\begin{aligned}
\left\langle-\nabla J_{0}(u), Y_{31}\right\rangle \geq & \frac{c}{\lambda_{h} \sum_{l \in I}\left|\nabla_{T} K\left(a_{l}\right)\right|} \\
& \times \sum_{i \in I}\left(8 c_{5} \frac{\left|\nabla_{T} K\left(a_{i}\right)\right|^{2}}{K_{1}\left(z_{k}\right)}+c_{2} \sum_{j \in I \cup \Gamma_{2}} \frac{\partial \varepsilon_{i j}}{\partial a_{i}} \nabla_{T} K\left(a_{i}\right)\right) \\
& +O\left(\sum_{i \in I, j \in \Gamma_{1}} \varepsilon_{i j}\right)+R
\end{aligned}
$$

Note that, for $i \neq j$, then $\partial \varepsilon_{i j} / \partial a_{i}=-\partial \varepsilon_{i j} / \partial a_{j}$, and therefore using the fact that, $z_{k}$ is a local maximum point, we have, for $i, j \in I$

$$
\begin{aligned}
& \frac{\partial \varepsilon_{i j}}{\partial a_{i}} \nabla_{T} K\left(a_{i}\right)+\frac{\partial \varepsilon_{j i}}{\partial a_{j}} \nabla_{T} K\left(a_{j}\right)=\frac{\partial \varepsilon_{i j}}{\partial a_{i}}\left(\nabla_{T} K\left(a_{i}\right)-\nabla_{T} K\left(a_{j}\right)\right) \\
& \quad=2 \lambda_{i} \lambda_{j}\left(a_{j}-a_{i}\right) \varepsilon_{i j}^{2}\left(D^{2} K\left(z_{k}\right)\left(a_{i}-a_{j}\right)+o\left(\left|a_{i}-z_{k}\right|+\left|a_{j}-z_{k}\right|\right)\right) \\
& \quad \geq 2 \lambda_{i} \lambda_{j}\left|a_{j}-a_{i}\right| \varepsilon_{i j}^{2} o\left(\left|a_{i}-z_{k}\right|+\left|a_{j}-z_{k}\right|\right) .
\end{aligned}
$$


But, since $\lambda_{i}, \lambda_{j}$ and $\lambda_{h}$ are of the same order, we get

$$
\begin{aligned}
\frac{\lambda_{i} \lambda_{j}\left|a_{j}-a_{i}\right| \varepsilon_{i j}^{2}}{\lambda_{h} \sum_{l \in I}\left|\nabla_{T} K\left(a_{l}\right)\right|} o\left(\left|a_{i}-z_{k}\right|+\left|a_{j}-z_{k}\right|\right) & =c \varepsilon_{i j}^{3 / 2} o\left(\frac{\left|a_{i}-z_{k}\right|+\left|a_{j}-z_{k}\right|}{\sum_{l \in I}\left|\nabla_{T} K\left(a_{l}\right)\right|}\right) \\
& =o\left(\varepsilon_{i j}^{3 / 2}\right) .
\end{aligned}
$$

Now, using the definition of $\Gamma_{2}$, for $i \in I, j \in \Gamma_{2}$, we have

$$
\varepsilon_{i j}=\frac{1}{\lambda_{i} \lambda_{j}\left|a_{i}-a_{j}\right|^{2}}+o\left(\varepsilon_{i j}\right) .
$$

Moreover, since $j \in \Gamma_{2}$ (which implies that $\lambda_{j} \geq \lambda_{h}$ and $\varepsilon_{i j} \leq c / \lambda_{j}$ ), an easy computation shows

$$
\begin{aligned}
\left|\frac{1}{\lambda_{h}} \frac{\partial \varepsilon_{i j}}{\partial a_{i}} \frac{\nabla_{T} K\left(a_{i}\right)}{\sum_{l \in I}\left|\nabla_{T} K\left(a_{l}\right)\right|}\right| & \leq c \frac{\sqrt{\lambda_{i} \lambda_{j}}}{\lambda_{h}} \varepsilon_{i j}^{3 / 2} \leq c \frac{\sqrt{\lambda_{i}}}{\lambda_{h}} \frac{1}{\lambda_{j}} \leq \frac{c}{\sqrt{M}} \frac{1}{\lambda_{h} \sqrt{\lambda_{j}}} \\
& =o\left(\frac{1}{\lambda_{h}^{3 / 2}}\right),
\end{aligned}
$$

where we will choose $M$ large enough. Thus, (4.16) becomes

$$
\begin{aligned}
\left\langle-\nabla J_{0}(u), Y_{31}\right\rangle \geq & c \sum_{i \in I} \frac{8 c_{5}\left|\nabla_{T} K\left(a_{i}\right)\right|^{2}}{K_{1}\left(z_{k}\right) \lambda_{h} \sum_{l \in I}\left|\nabla_{T} K\left(a_{l}\right)\right|}+o\left(\frac{1}{\lambda_{h}^{3 / 2}}\right) \\
& +O\left(\sum_{i \in I, j \in \Gamma_{1}} \varepsilon_{i j}\right)+R \\
\geq & \frac{c}{\sharp I} \frac{8 c_{5}\left|\nabla_{T} K\left(a_{h}\right)\right|}{K_{1}\left(z_{k}\right) \lambda_{h}}+o\left(\frac{1}{\lambda_{h}^{3 / 2}}\right)+O\left(\sum_{i \in I, j \in \Gamma_{1}} \varepsilon_{i j}\right)+R,
\end{aligned}
$$

where $\sharp I$ denotes the cardinal of $I$. Note that, there exists $i_{0} \in I$, such that $\left|\nabla_{T} K\left(a_{h}\right)\right| \geq \gamma\left|a_{h}-a_{i_{0}}\right| / 2, \quad$ where $\gamma$ is defined in assumption $\left(H_{2}\right)$,

and from (4.15), we have $\varepsilon_{h i_{0}} \leq c / \lambda_{i_{0}}$, which implies that $\left(\lambda_{h} \lambda_{i_{0}} \mid a_{h}-\right.$ $\left.\left.a_{i_{0}}\right|^{2}\right)^{-1} \leq c / \lambda_{i_{0}}$. Hence, $\left|a_{h}-a_{i_{0}}\right| \geq c / \sqrt{\lambda_{h}}$. Therefore, using again (4.19), (4.20) and the definition of $\Gamma_{2}$, we get

$$
\begin{gathered}
\left\langle-\nabla J_{0}(u), Y_{31}\right\rangle \geq c \sum_{i \leq q} \frac{1}{\lambda_{i}^{3 / 2}}+c \sum_{i \in \Gamma_{2}, j \neq i} \varepsilon_{i j}^{3 / 2}+c \sum_{i, j \in I, i \neq j} \varepsilon_{i j}^{3 / 2} \\
+O\left(\sum_{i \in I, j \in \Gamma_{1}} \varepsilon_{i j}\right)+R .
\end{gathered}
$$


It remains to make appear the $\varepsilon_{i j}$ for $i \in \Gamma_{1}$. For this aim, we define the following vector fields

$$
Y_{32}=-\sum_{i \in \Gamma_{1}} \lambda_{i} \frac{\partial \delta_{i}}{\partial \lambda_{i}} 2^{i} \quad \text { and } \quad Y_{33}=\sum_{i \leq q} \psi\left(\frac{\lambda_{i}}{C}\left(1-J_{0}(u)^{2} \alpha_{i}^{2} K\left(a_{i}\right)\right)\right) \delta_{i}
$$

where $C$ is a large positive constant and $\psi$ is defined in (4.3). As in the proof of (4.12), we get

$$
\left\langle-\nabla J_{0}(u), Y_{32}\right\rangle \geq c \sum_{i \in \Gamma_{1}}\left(\sum_{j \neq i} \varepsilon_{i j}+\frac{1}{\lambda_{i}}\right)+O\left(\sum_{k \neq r} \varepsilon_{k r}^{2}+\sum_{i \leq q} \frac{1}{\lambda_{i}^{2}}\right) .
$$

Using also the Appendix 2 [Proposition 7.2] and the definition of $\psi$, we derive

$$
\begin{aligned}
&\left\langle\nabla J_{0}(u), Y_{33}\right\rangle \geq \sum_{i \leq q}\left|\psi\left(\frac{\lambda_{i}}{C}\left(1-J_{0}(u)^{2} \alpha_{i}^{2} K\left(a_{i}\right)\right)\right)\right| \\
& {\left[\left|1-J_{0}(u)^{2} \alpha_{i}^{2} K\left(a_{i}\right)\right|+O\left(\frac{1}{\lambda_{i}}+\sum_{j \neq i} \varepsilon_{i j}\right)\right] . }
\end{aligned}
$$

Observe that, choosing $C$ large enough, if for some indices $i$ we have

$$
\left.\psi\left(\frac{\lambda_{i}}{C}\left(1-J_{0}(u)^{2} \alpha_{i}^{2} K\left(a_{i}\right)\right)\right) \neq 0, \text { then } \frac{1}{\lambda_{i}}=o\left(\mid 1-J_{0}(u)^{2} \alpha_{i}^{2} K\left(a_{i}\right)\right) \mid\right) .
$$

In the other case, we have: $\left.\mid 1-J_{0}(u)^{2} \alpha_{i}^{2} K\left(a_{i}\right)\right) \mid=O\left(\lambda_{i}^{-1}\right)$. Hence, we can make appear this term from $\lambda_{i}^{-3 / 2}$.

Our vector field in this case is $Y_{k}^{3}=\sqrt{M^{\prime \prime}} Y_{31}+M^{\prime \prime} Y_{32}+Y_{33}$, where $M^{\prime \prime}$ is a large positive constant. By (4.21)-(4.23), we obtain

$$
\left\langle-\nabla J_{0}(u), Y_{k}^{3}\right\rangle \geq c \sum_{i \leq q}\left(\sum_{j \neq i} \varepsilon_{i j}^{3 / 2}+\frac{1}{\lambda_{i}^{3 / 2}}+\left|1-J_{0}(u)^{2} \alpha_{i}^{2} K\left(a_{i}\right)\right|^{3 / 2}\right) .
$$

Case 4. $I$ contains at least two indices, $z_{k}$ is not a local maximum point of $K_{1}$ and for all $j \in I$, (4.15) holds.

We introduce the subset $I^{\prime}=\left\{i \in I: \lambda_{q}\left|\nabla_{T} K\left(a_{i}\right)\right|>\bar{d}\right\}$ where $\bar{d}$ is a small positive constant.

Observe that $I \backslash I^{\prime}$ contains at most one point. In this case, two subcases may occur.

Case 4.1. $I=I^{\prime}$. We define the vector field

$$
Y_{41}^{1}=\sum_{i \in I} \frac{1}{\lambda_{1}} \frac{\partial \delta_{i}}{\partial a_{i}} \frac{\nabla_{T} K\left(a_{i}\right)}{\left|\nabla_{T} K\left(a_{i}\right)\right|}
$$


Thus, using the Appendix 2 [Proposition 7.2], we get

$$
\begin{aligned}
\left\langle-\nabla J_{0}(u), Y_{41}^{1}\right\rangle \geq & \frac{c}{\lambda_{1}} \sum_{i \in I}\left(8 c_{5} \frac{\left|\nabla_{T} K\left(a_{i}\right)\right|}{K_{1}\left(z_{k}\right)}(1+o(1))+c_{2} \sum_{l \in I} \frac{\partial \varepsilon_{l i}}{\partial a_{i}} \frac{\nabla_{T} K\left(a_{i}\right)}{\left|\nabla_{T} K\left(a_{i}\right)\right|}\right. \\
& \left.+O\left(\frac{1}{\lambda_{1}} \sum_{l \in \Gamma_{2}}\left|\frac{\partial \varepsilon_{l i}}{\partial a_{i}}\right|\right)\right)+O\left(\sum_{i \in I, j \in \Gamma_{1}} \varepsilon_{i j}\right)+R .
\end{aligned}
$$

First observe that, for $i \in I$ and $l \in \Gamma_{2}$, using (4.18) and (4.15), we get

$$
\begin{aligned}
\left|\frac{\partial \varepsilon_{i l}}{\partial a_{i}}\right| & \leq \frac{c \varepsilon_{i l}}{\left|a_{i}-a_{l}\right|} \leq \frac{c}{\lambda_{l}\left|a_{i}-a_{l}\right|} \leq \frac{1}{\sqrt{M}} \frac{c}{\sqrt{\lambda_{i} \lambda_{l}}\left|a_{i}-a_{l}\right|} \leq \frac{c \sqrt{\varepsilon_{i l}}}{\sqrt{M}} \leq \frac{c}{\sqrt{M \lambda_{l}}} \\
& \leq \frac{c}{M} \frac{1}{\sqrt{\lambda_{i}}} .
\end{aligned}
$$

Moreover, from (4.15), we get that for $i, j \in I$,

$$
\begin{aligned}
& \max \left(\frac{1}{\lambda_{i}}, \frac{1}{\lambda_{j}}\right) \frac{1+o(1)}{\left|a_{i}-a_{j}\right|^{2}}<\frac{1}{c_{2}} \frac{1}{1-\varrho} \frac{4 c_{3}}{K_{1}\left(z_{k}\right)} \frac{\partial K}{\partial \nu}\left(z_{k}\right):=\gamma_{k}, \\
& \left|a_{i}-a_{j}\right| \geq \gamma_{k}^{-1 / 2} \max \left(\lambda_{i}^{-1}, \lambda_{j}^{-1}\right)^{1 / 2} \geq \frac{1}{2} \gamma_{k}^{-1 / 2}\left(\lambda_{i}^{-1 / 2}+\lambda_{j}^{-1 / 2}\right)(1+o(1)), \\
& \left|\nabla_{T} K\left(a_{i}\right)\right|+\left|\nabla_{T} K\left(a_{j}\right)\right| \geq \gamma\left|a_{i}-a_{j}\right| \geq \frac{1}{2} \gamma \gamma_{k}^{-1 / 2}\left(\lambda_{i}^{-1 / 2}+\lambda_{j}^{-1 / 2}\right)(1+o(1)) .
\end{aligned}
$$

Thus we get

$$
\sum_{i \in I}\left|\nabla_{T} K\left(a_{i}\right)\right| \geq \frac{1}{2} \gamma \gamma_{k}^{-1 / 2}(1+o(1)) \sum_{i \in I} \frac{1}{\lambda_{i}^{1 / 2}} .
$$

Now, observe that, using (4.15) and (4.27), we have

$$
\begin{aligned}
\sum_{l \in I, l \neq i}\left|\frac{\partial \varepsilon_{i l}}{\partial a_{i}}\right| & =\sum_{l \in I, l \neq i} \frac{(1+o(1))}{\left|a_{i}-a_{j}\right|} \varepsilon_{i l} \leq(1+o(1)) \gamma_{k}^{1 / 2} \sqrt{\lambda_{i}} \sum_{l \in I, l \neq i} \varepsilon_{i l} \\
& \leq \frac{\gamma_{k}^{3 / 2}}{\sqrt{\lambda_{i}}}\left(\frac{1}{c_{2}}+o(1)\right) .
\end{aligned}
$$

Combining (4.29), (4.30), (4.25) and (4.26), we get

$$
\begin{aligned}
\left\langle-\nabla J_{0}(u), Y_{41}^{1}\right\rangle \geq & \frac{c}{\lambda_{1}} \gamma_{k}^{-1 / 2} \sum_{i \in I} \frac{1}{\sqrt{\lambda_{i}}}\left(\gamma \frac{4 c_{5}}{K_{1}\left(z_{k}\right)}-\gamma_{k}^{2}+O\left(\frac{1}{M}\right)\right) \\
& +O\left(\sum_{i \in I, j \in \Gamma_{1}} \varepsilon_{i j}\right)+R .
\end{aligned}
$$

From the assumption $\left(\mathrm{H}_{2}\right)$, we can choose $\varrho$ so that

$$
\frac{\gamma_{k}^{2}}{1-\varrho}<\gamma \frac{4 c_{5}}{K_{1}\left(z_{k}\right)} \text {. }
$$


Therefore

$$
\begin{aligned}
& \left\langle-\nabla J_{0}(u), Y_{41}^{1}\right\rangle \geq c \sum_{i \leq q} \frac{1}{\lambda_{i}^{3 / 2}}+c \sum_{i \in \Gamma_{2}, j \neq i} \varepsilon_{i j}^{3 / 2}+c \sum_{i, j \in I, i \neq j} \varepsilon_{i j}^{3 / 2} \\
& +O\left(\sum_{i \in I, j \in \Gamma_{1}} \varepsilon_{i j}\right)+R \text {. }
\end{aligned}
$$

Case 4.2. $I \neq I^{\prime}$. We define the vector field

$$
Y_{41}^{2}=\sum_{i \in I^{\prime}} \frac{1}{\lambda_{1}} \frac{\partial \delta_{i}}{\partial a_{i}} \frac{\nabla_{T} K\left(a_{i}\right)}{\left|\nabla_{T} K\left(a_{i}\right)\right|} .
$$

Let $i_{0} \in I \backslash I^{\prime}$, using the first part of (4.28), we get : $\left|\nabla_{T} K\left(a_{i}\right)\right| \geq \gamma\left|a_{i}-a_{i_{0}}\right| / 2$ for each $i \in I^{\prime}$.

Using the Appendix 2 [Proposition 7.2], we get

$$
\begin{aligned}
\langle- & \left.\nabla J_{0}(u), Y_{41}^{2}\right\rangle \\
\geq & \frac{c}{\lambda_{1}} \sum_{i \in I^{\prime}}\left(8 c_{5} \frac{\left|\nabla_{T} K\left(a_{i}\right)\right|}{K_{1}\left(z_{k}\right)}+c_{2} \sum_{l \in I} \frac{\partial \varepsilon_{l i}}{\partial a_{i}} \frac{\nabla_{T} K\left(a_{i}\right)}{\left|\nabla_{T} K\left(a_{i}\right)\right|}\right. \\
& \left.+O\left(\sum_{l \in \Gamma_{2}}\left|\frac{\partial \varepsilon_{l i}}{\partial a_{i}}\right|+\lambda_{1} \sum_{j \in \Gamma_{1}} \varepsilon_{i j}\right)\right)+R \\
\geq & \frac{c}{\lambda_{1}} \sum_{i \in I^{\prime}}\left(\frac{4 c_{5} \gamma}{K_{1}\left(z_{k}\right)}\left|a_{i}-a_{i_{0}}\right|+c_{2} \sum_{l \in I} \frac{\partial \varepsilon_{l i}}{\partial a_{i}} \frac{\nabla_{T} K\left(a_{i}\right)}{\left|\nabla_{T} K\left(a_{i}\right)\right|}\right. \\
& \left.+O\left(\sum_{l \in \Gamma_{2}}\left|\frac{\partial \varepsilon_{l i}}{\partial a_{i}}\right|+\lambda_{1} \sum_{j \in \Gamma_{1}} \varepsilon_{i j}\right)\right)+R .
\end{aligned}
$$

From (4.30) and the same arguments than the case 4.1, we derive

$$
\begin{aligned}
& \left\langle-\nabla J_{0}(u), Y_{41}^{2}\right\rangle \geq c \sum_{i \leq q} \frac{1}{\lambda_{i}^{3 / 2}}+c \sum_{i \in \Gamma_{2}, j \neq i} \varepsilon_{i j}^{3 / 2}+c \sum_{i, j \in I, i \neq j} \varepsilon_{i j}^{3 / 2} \\
& +O\left(\sum_{i \in I^{\prime}, j \in \Gamma_{1}} \varepsilon_{i j}\right)+R .
\end{aligned}
$$

Now, let us define the vector field $Y_{41}$ by a convex combination of $Y_{41}^{1}$ and $Y_{41}^{2}$. To make appear the $\varepsilon_{i j}$ for $i \in \Gamma_{1}$, we define the same vector fields in the last case

$$
Y_{42}=-\sum_{i \in \Gamma_{1}} \lambda_{i} \frac{\partial \delta_{i}}{\partial \lambda_{i}} 2^{i} \quad \text { and } \quad Y_{43}=\sum_{i \leq q} \psi\left(\frac{\lambda_{i}}{C}\left(1-J_{0}(u)^{2} \alpha_{i}^{2} K\left(a_{i}\right)\right)\right) \delta_{i},
$$

where $C$ is a large positive constant. Our vector field in this case is $Y_{k}^{4}=$ $\sqrt{M_{4}} Y_{41}+M_{4} Y_{42}+Y_{43}$, where $M_{4}^{\prime}$ is a large positive constant. By (4.32), (4.33), (4.22) and (4.23), we have 


$$
\left\langle-\nabla J_{0}(u), Y_{k}^{4}\right\rangle \geq c \sum_{i \leq q}\left(\sum_{j \neq i} \varepsilon_{i j}^{3 / 2}+\frac{1}{\lambda_{i}^{3 / 2}}+\left|1-J_{0}(u)^{2} \alpha_{i}^{2} K\left(a_{i}\right)\right|^{3 / 2}\right) .
$$

Note that, we separated the cases 3 and 4 , because (4.17) does not hold if $z_{k}$ is not a local maximum point of $K_{1}$. Moreover, (1.3) is not assumed if $z_{k}$ is a local maximum point.

Let $Y_{k}$ be a convex combination of $Y_{k}^{1}-Y_{k}^{4}$. Using (4.9), (4.14), (4.24) and (4.34), we get

$$
\left\langle-\nabla J_{0}(u), Y_{k}\right\rangle \geq c \sum_{i \leq q}\left(\sum_{j \neq i} \varepsilon_{i j}^{3 / 2}+\frac{1}{\lambda_{i}^{3 / 2}}+\left|1-J_{0}(u)^{2} \alpha_{i}^{2} K\left(a_{i}\right)\right|^{3 / 2}\right) .
$$

It is easy to see that $\dot{\lambda}_{\max } \leq 0$ and $\left\|Y_{k}\right\|$ is bounded. Hence the proof is completed.

Proof of Proposition 4.2. Let

$$
u=\sum_{i \leq q} \alpha_{i} \delta_{\left(a_{i}, \lambda_{i}\right)}+\sum_{i>q} \alpha_{i} P \delta_{\left(a_{i}, \lambda_{i}\right)} \in V(p, \varepsilon) .
$$

Without loss of generality, we can assume that $\lambda_{1} \leq \cdots \leq \lambda_{q}$ and $\lambda_{q+1} \leq$ $\cdots \leq \lambda_{p}$.

Since $\left|\partial K\left(z_{i}\right) / \partial \nu\right|>0$ for any critical point $z_{i}$ of $K_{1}$, there exist $\mu>0$ and $c>0$ such that $|\partial K(a) / \partial \nu|>c$ for any $a \in \cup B\left(z_{i}, 2 \mu\right)$. In the sequel, we will choose $\mu$ such that $B\left(z_{i}, 8 \mu\right) \cap B\left(z_{j}, 8 \mu\right)=\phi$ for each $i \neq j$.

As said before, the construction is based on moving the points $a_{i}$ if they are away from the critical points of $K$ or $K_{1}$. In the other cases the vector field is basically defined on the variables $\lambda_{i}$. For this raison, we divide $V(p, \varepsilon)$ into some regions $V_{i}(p, \varepsilon)$, and we will build a vector field $W_{i}$ on each $V_{i}(p, \varepsilon)$. Our global vector field $W$ will be built using a convex combination of the vector fields $W_{i}$. For $k \leq r_{1}$, we introduce the set

$$
\begin{gathered}
L_{k}:=\left\{i \leq q / \exists i_{1}=i, \ldots, i_{j}, \text { s.t. } a_{i_{j}} \in B\left(z_{k}, \mu\right) \text { and } d\left(a_{i_{l}}, a_{i_{l-1}}\right)<\mu / p,\right. \\
\forall l \leq j\} .
\end{gathered}
$$

It is clear that if $i \in L_{k}$ and $j \notin L_{k}$ we have $\left|a_{i}-a_{j}\right| \geq c>0$. Let

$$
\begin{aligned}
V_{1}(p, \varepsilon):= & \left\{u \in V(p, \varepsilon): \forall i, a_{i} \in \cup_{k \leq r_{1}} B\left(z_{k}, 2 \mu\right) \cup \cup_{k} B\left(y_{k}, 2 \mu\right)\right. \\
& \text { and } \left.\sharp L_{k} \leq 1, \forall k \leq r_{1}\right\} . \\
V_{2}(p, \varepsilon):= & \left\{u \in V(p, \varepsilon): \exists i_{0}, a_{i_{0}} \notin \cup_{k \leq r_{1}} B\left(z_{k}, 2 \mu\right) \cup \cup_{k} B\left(y_{k}, 2 \mu\right)\right. \\
& \text { and } \left.\sharp L_{k} \leq 1, \forall k \leq r_{1}\right\} . \\
V_{3}(p, \varepsilon):= & \left\{u \in V(p, \varepsilon): \exists k \leq r_{1}, \text { such that } \sharp L_{k} \geq 2\right\} .
\end{aligned}
$$

Recall that $r_{1}$ satisfies : $\partial K\left(z_{k}\right) / \partial \nu>0$ for any critical point $z_{k}$ of $K_{1}$ such that $k \leq r_{1}$. 


\subsection{Construction of $W_{1}$ on $V_{1}(p, \varepsilon)$}

Let $\bar{u}:=\sum_{i>q} \alpha_{i} P \delta_{i}$. It is clear that $\bar{u} \in V(p-q, \varepsilon)$ and all the concentration points are in $S_{+}^{4}$. Thus, we can apply the vector field (denoted here $Z_{1}$ ) defined in Proposition 3.1 of [10] (see also [7]). Then, we get

$$
\left\langle-\nabla J_{0}(u), Z_{1}\right\rangle \geq c \sum_{i>q}\left(\sum_{j>q, j \neq i} \varepsilon_{i j}+\frac{1}{\left(\lambda_{i} d_{i}\right)^{2}}+\frac{\left|\nabla K\left(a_{i}\right)\right|}{\lambda_{i}}\right)+O\left(\sum_{j \leq q} \varepsilon_{i j}\right) .
$$

It is interesting to mention that, along the flow lines of $Z_{1}$, the only case where the maximum of the $\lambda_{i}$ 's is not bounded is when each point $a_{i}$ is close to a critical point $y_{j_{i}}$ of $K$ with $j_{i} \neq j_{k}$ for $i \neq k$ and $\rho\left(y_{j_{1}}, \ldots, y_{j_{s}}\right)>0$, where $s=p-q$ and $\rho\left(y_{j_{1}}, \ldots, y_{j_{s}}\right)$ denotes the least eigenvalue of $M\left(y_{j_{1}}, \ldots, y_{j_{s}}\right)$.

For the indices $i \leq q$, we introduce the sets

$$
P_{1}=\left\{i \leq q: \lambda_{i} \geq m^{\prime} \lambda_{q+1}^{2}\right\} \quad \text { and } \quad P_{2}=\left\{i \leq q: i \notin P_{1}\right\},
$$

where $m^{\prime}$ is a small positive constant. Note that, if $p=q$ then $P_{1}=\emptyset$ and $P_{2}=\{1, \ldots, p\}$.

We remark that for $i \in P_{1}$, we can make appear the variables $\lambda_{i}^{-1}$ and $\varepsilon_{i j}$ from $\lambda_{q+1}^{-2}$. In fact, for each $i \neq j$, we have $\left|a_{i}-a_{j}\right| \geq c>0$ and therefore,

$$
\lambda_{i}^{-1}=O\left(\lambda_{q+1}^{-2}\right), \quad \text { and } \quad \varepsilon_{i j}=\frac{1}{\lambda_{i} \lambda_{j}\left|a_{i}-a_{j}\right|^{2}}+o\left(\varepsilon_{i j}\right)=o\left(\lambda_{q+1}^{-2}\right) .
$$

It remains to make appear the indices $i \in P_{2}$. For this goal, we define

$$
Z_{2}=\sum_{i \in P_{2}} \lambda_{i} \frac{\partial \delta_{i}}{\partial \lambda_{i}}
$$

Note that, for $i \in P_{2}$, it is easy to obtain that $\varepsilon_{i j}=o\left(1 / \lambda_{i}\right)$ for each $j \neq i$. Hence, using the Appendix 2 [Proposition 7.2], we get

$$
\left\langle-\nabla J_{0}(u), Z_{2}\right\rangle \geq c \sum_{i \in P_{2}}\left(\frac{1}{\lambda_{i}}+\sum_{j \neq i} \varepsilon_{i j}\right)+O\left(\sum_{k \neq r} \varepsilon_{k r}^{2}\right) .
$$

Now, we define the vector field $Z_{3}=Z_{1}+Z_{2}$. Combining (4.35), (4.36) and (4.37), we get

$$
\left\langle-\nabla J_{0}(u), Z_{3}\right\rangle \geq c\left(\sum_{j \neq i} \varepsilon_{i j}+\sum_{i>q}\left(\frac{1}{\left(\lambda_{i} d_{i}\right)^{2}}+\frac{\left|\nabla K\left(a_{i}\right)\right|}{\lambda_{i}}\right)+\sum_{i \leq q} \frac{1}{\lambda_{i}}\right) .
$$

To add the other variables which appear in Proposition 4.2, we introduce the vector field

$$
Z_{4}=\sum_{i \leq q} Z_{i}^{\alpha}, \quad \text { where } Z_{i}^{\alpha} \text { is defined by (4.2). }
$$

Our vector field $W_{1}$ will be the following

$$
W_{1}=M_{1} Z_{3}+Z_{4} \text {, where } M_{1} \text { is a large positive constant. }
$$


By (4.38) and (4.4), we obtain the estimate of Claim $(i)$ for $W_{1}$ instead of $W$. Note that the only case where the maximum of the $\lambda_{i}$ 's for $i \leq q$ is not bounded is when $\rho\left(y_{j_{1}}, \ldots, y_{j_{s}}\right)>0$, where $s=p-q$ or when all the points $a_{i}$ are in the boundary $(q=p)$.

\subsection{Construction of $W_{2}$ on $V_{2}(p, \varepsilon)$}

Let $P=\cup_{k \leq r_{1}} L_{k}$. We can write $u:=u_{1}+u_{2}$, where $u_{1}=\sum_{i \in P} \alpha_{i} \delta_{i}$ and $u_{2}=u-u_{1}$. Observe, if $i \notin P$ then $a_{i} \notin \cup_{k \leq r_{1}} B\left(z_{k}, 2 \mu\right)$ or $a_{i} \in S_{+}^{4}$. Note that $u_{2} \in V(p-\sharp P, \varepsilon)$. Hence, we can use the vector field (denoted here $Z_{5}$ ) defined in $[10]$ (we apply $Z_{5}$ only to $u_{2}$ ). Then, we get

$$
\begin{aligned}
\left\langle-\nabla J_{0}(u), Z_{5}\right\rangle \geq & c \sum_{i \leq q, i \notin P}\left(\sum_{j \neq i} \varepsilon_{i j}+\frac{1}{\lambda_{i}}+\left|1-J_{0}(u)^{2} \alpha_{i}^{2} K\left(a_{i}\right)\right|\right) \\
& +c \sum_{i>q}\left(\sum_{j \notin P, j \neq i} \varepsilon_{i j}+\frac{1}{\left(\lambda_{i} d_{i}\right)^{2}}+\frac{\left|\nabla K\left(a_{i}\right)\right|}{\lambda_{i}}+O\left(\sum_{j \in P} \varepsilon_{i j}\right)\right),
\end{aligned}
$$

where we use $\varepsilon_{i j}=o\left(\lambda_{i}^{-1}\right)$ since we have $\left|a_{i}-a_{j}\right| \geq c$ for $i \leq q, i \notin P$ and $j \in P$.

Note that, there exists $i_{0}$ such that $a_{i_{0}} \notin \cup_{k \leq r_{1}} B\left(z_{k}, \mu\right) \cup \cup_{k} B\left(y_{k}, \mu\right)$, this implies that $c \lambda_{i_{0}}^{-1}$ is in the lower bound of (4.39). Furthermore, the maximum of the $\lambda_{i}$ 's, $i \notin P$, is bounded along the flow lines generated by $Z_{5}$ (see Proposition 3.1 of [10] for more details).

To make appear the other variables with indices in $P$, we need to introduce the following set

$$
P^{\prime}=\left\{i \in P: \quad M_{2} \lambda_{i}<\lambda_{i_{0}}\right\}, \quad \text { where } M_{2} \text { is a large positive constant. }
$$

We remark that, for $i \in P \backslash P^{\prime}$, we can make appear $\lambda_{i}^{-1}$ 's and $\varepsilon_{i j}$ 's from $\lambda_{i_{0}}^{-1}$. In fact we have

$$
\frac{1}{\lambda_{i}}=O\left(\frac{1}{\lambda_{i_{0}}}\right), \quad \text { and } \quad \varepsilon_{i j}=\frac{1+o(1)}{\lambda_{i} \lambda_{j}\left|a_{i}-a_{j}\right|^{2}}=o\left(\frac{1}{\lambda_{i_{0}}}\right), \quad \text { where } i \in P \backslash P^{\prime} .
$$

It remains to make appear the indices $i \in P^{\prime}$. For this aim, we will increase the $\lambda_{i}$ 's, $i \in P^{\prime}$ (note that for $i \in P^{\prime}$, we have $\lambda_{i} \leq M_{2}^{-1} \lambda_{i_{0}}$ ).

$$
Z_{6}=\sum_{i \in P^{\prime}} \lambda_{i} \frac{\partial \delta_{i}}{\partial \lambda_{i}}, \quad Z_{7}=\sum_{i \leq q} Z_{i}^{\alpha}, \quad \text { where } Z_{i}^{\alpha} \text { is defined by (4.2). }
$$

Note that, for $i \in P^{\prime}$, it is easy to obtain that $\varepsilon_{i j}=o\left(1 / \lambda_{i}\right)$ for each $j \neq i$ (since $\left|a_{i}-a_{j}\right| \geq c$ ). Hence, using the Appendix 2 [Proposition 7.2], we get

$$
\left\langle-\nabla J_{0}(u), Z_{6}\right\rangle \geq c \sum_{i \in P^{\prime}}\left(\frac{1}{\lambda_{i}}+\sum_{j \neq i} \varepsilon_{i j}\right)+O\left(\sum_{k \neq r} \varepsilon_{k r}^{2}\right) .
$$


Our vector field $W_{2}$ will be the following

$W_{2}=M_{2}^{\prime} Z_{5}+\sqrt{M_{2}^{\prime}} Z_{6}+Z_{7}$, where $M_{2}^{\prime}$ is a large positive constant.

Combining (4.39)-(4.41) and (4.4), we obtain the estimate of Claim $(i)$ for $W_{2}$ instead of $W$.

\subsection{Construction of $W_{3}$ on $V_{3}(p, \varepsilon)$}

We introduce the following sets

$P_{k}:=\left\{i \in L_{k}: \quad\right.$ s.t. $\left.\sharp L_{k} \geq 2\right\}, \quad P:=\left\{i \leq q: i \in \cup_{k \leq r_{1}} L_{k}\right.$ and $\left.i \notin \cup_{k} P_{k}\right\}$.

In this case, we can write $u$ as $u=\sum_{k} u_{1}^{k}+u_{2}+u_{3}$, where $u_{1}^{k}=\sum_{i \in P_{k}} \alpha_{i} \delta_{i}$ and $u_{2}=\sum_{i \in P} \alpha_{i} \delta_{i}$. Hence, $u_{1}^{k} \in V\left(\sharp P_{k}, \varepsilon, k\right)$ and we can apply Theorem 1.2 for each $u_{1}^{k}$. Thus, there exists $Z_{8}=\sum_{k} Y_{k}$ which moves only the variables indexed by $i \in \cup_{k} P_{k}$ and we obtain

$$
\begin{aligned}
\left\langle-\nabla J_{0}(u), Z_{8}\right\rangle \geq & c \sum_{i \in \cup P_{k}}\left(\sum_{j \in \cup P_{k}} \varepsilon_{i j}^{3 / 2}+\frac{1}{\lambda_{i}^{3 / 2}}+\left|1-J_{0}(u)^{2} \alpha_{i}^{2} K\left(a_{i}\right)\right|^{3 / 2}\right. \\
& \left.+O\left(\sum_{j \notin \cup P_{k}} \varepsilon_{i j}\right)\right)
\end{aligned}
$$

where we have used $\varepsilon_{i j}=O\left(\lambda_{i}^{-2}+\lambda_{j}^{-2}\right)$ since we have $\left|a_{i}-a_{j}\right| \geq c>0$ for $i \in P_{k}$ and $j \notin P_{k}$.

Let $k_{0}=\min \left\{i: i \in \cup_{k} P_{k}\right\}$, we introduce the set

$$
P^{\prime}=\left\{i \in P: \quad M_{3} \lambda_{i}<\lambda_{k_{0}}\right\} \quad \text { where } M_{3} \text { is a large positive constant. }
$$

We remark that, for $i, j \in P \backslash P^{\prime}$, we have $\left|a_{i}-a_{j}\right| \geq c>0$, and therefore we can make appear the variables $\lambda_{i}^{-3 / 2}$ and $\varepsilon_{i j}^{3 / 2}$ from $\lambda_{k_{0}}^{-3 / 2}$. We define also the following vector field

$$
\begin{aligned}
Z_{9} & =\sum_{i \in P \backslash P^{\prime}} \psi\left(\frac{\lambda_{i}}{C^{\prime}}\left(1-J_{0}(u)^{2} \alpha_{i}^{2} K\left(a_{i}\right)\right)\right) \delta_{i} \quad \text { and } \\
Z_{10} & =\sum_{i \in P^{\prime}}\left(M_{3}^{\prime} \lambda_{i} \frac{\partial \delta_{i}}{\partial \lambda_{i}}+Z_{i}^{\alpha}\right)
\end{aligned}
$$

where $C^{\prime}$ and $M_{3}^{\prime}$ are large positive constants and $Z_{i}^{\alpha}$ is defined by (4.2).

Note that, for $i \in P^{\prime}$, it is easy to obtain that, $\left|a_{i}-a_{j}\right| \geq c>0$, for $i \neq j$ and therefore $\varepsilon_{i j}=o\left(1 / \lambda_{i}\right)$ for each $j \neq i$. Using the Appendix 2 [Proposition 7.2], we get

$$
\begin{aligned}
\left\langle-\nabla J_{0}(u), Z_{10}\right\rangle \geq & c \sum_{i \in P^{\prime}}\left(\frac{1}{\lambda_{i}}+\sum_{j \leq q} \varepsilon_{i j}+\left|1-J_{0}(u)^{2} \alpha_{i}^{2} K\left(a_{i}\right)\right|\right. \\
& \left.+O\left(\sum_{j>q} \varepsilon_{i j}+\sum_{k \neq r} \varepsilon_{k r}^{2}\right)\right) .
\end{aligned}
$$


For $u_{3}$, we can use the vector field (denoted here $Z_{11}$ ) defined in Proposition 3.1 of $[10]$. (we will apply $Z_{11}$ only to $u_{3}$ ). Then, we get

$$
\begin{aligned}
\left\langle-\nabla J_{0}(u), Z_{11}\right\rangle \geq c & \sum_{i \leq q, i \notin\left(\cup_{k} L_{k}\right)}\left(\sum_{j \neq i} \varepsilon_{i j}+\frac{1}{\lambda_{i}}+\left|1-J_{0}(u)^{2} \alpha_{i}^{2} K\left(a_{i}\right)\right|\right) \\
& +c \sum_{i>q}\left(\sum_{j \notin\left(\cup_{k} L_{k}\right), j \neq i} \varepsilon_{i j}+\frac{1}{\left(\lambda_{i} d_{i}\right)^{2}}+\frac{\left|\nabla K\left(a_{i}\right)\right|}{\lambda_{i}}\right. \\
& \left.+O\left(\sum_{j \in \cup_{k} L_{k}} \varepsilon_{i j}\right)\right),
\end{aligned}
$$

where we use $\varepsilon_{i j}=o\left(\lambda_{i}^{-1}\right)$ since we have $\left|a_{i}-a_{j}\right| \geq c>0$ for $i \leq q, i \notin \cup_{k} L_{k}$ and $j \in \cup_{k} L_{k}$.

Now, we see that there exists $d_{0}>0$ such that for any a satisfying $d_{a}=$ $d\left(a, \partial S_{+}^{4}\right) \leq d_{0}$, we have

$$
H(a, a) \geq M_{0}|\Delta K(a)| \quad \text { and } \quad H(a, a) \sim d_{a}^{-2} / 4,
$$

where $M_{0}$ is a large positive constant. We need to introduce the subset of points which are close to the boundary, for that purpose, let us introduce the following set

$$
\begin{gathered}
F_{0}:=\left\{i>q / \exists i_{1}=i, \ldots, i_{l}, \text { s.t. } d\left(a_{i_{l}}, \partial S_{+}^{4}\right)<d_{0} / p \text { and } d\left(a_{i_{j}}, a_{i_{j-1}}\right)<d_{0} / p,\right. \\
\forall j \leq l\} .
\end{gathered}
$$

It is easy to see that the following claims holds :

- if $i \in F_{0}, j \notin F_{0}, j \geq q+1$, we have $d\left(a_{i}, a_{j}\right) \geq d_{0} / p$

- for any $i \in F_{0}$, we have $H\left(a_{i}, a_{i}\right) \geq M_{0}\left|\Delta K\left(a_{i}\right)\right|$ and $H\left(a_{i}, a_{i}\right) \sim 1 /\left(4 d_{i}^{2}\right)$

- for any $j \notin F_{0}$ and $j \geq q+1$, we have $d_{j}>d_{0} / p$ and $\varepsilon_{i j}=O\left(\lambda_{i}^{-2}\right)+o\left(\lambda_{j}^{-2}\right)$ if $i \leq q$.

Let

$$
Z_{12}=-\sum_{i \in F_{0}} \lambda_{i} \frac{\partial P \delta_{i}}{\partial \lambda_{i}} 2^{i}
$$

Using the Appendix 2 [Proposition 7.3], we get

$$
\left\langle-\nabla J_{0}(u), Z_{12}\right\rangle \geq c \sum_{i \in F_{0}}\left(\sum_{j \neq i} \varepsilon_{i j}+\frac{1}{\left(\lambda_{i} d_{i}\right)^{2}}\right)+O\left(\sum_{k \neq r} \varepsilon_{k r}^{2}\right) .
$$

Our vector field $W_{3}$ will be the following

$W_{3}=Z_{8}+Z_{9}+Z_{10}+Z_{11}+M_{4} Z_{12}$, where $M_{4}$ is a large positive constant. 
Combining (4.42)-(4.45) and (4.23), we obtain the estimate of Claim $(i)$ for $W_{3}$ instead of $W$.

Finally, we use a convex combination of $W_{1}, W_{2}$ and $W_{3}$ to build our global vector field $W$ in $V(p, \varepsilon)$ and the estimate $(i)$ follows.

We can obtain the estimate $(i i)$, arguing as in Appendix B of [7], using the estimates $(i),\left\|\nabla J_{0}(u+\bar{v})\right\|$ and $\|\bar{v}\|$.

Observe that in $V_{2}(p, \varepsilon)$ and $V_{3}(p, \varepsilon)$ the maximum of $\lambda_{i}$ 's is always a decreasing function along the flow lines. In $V_{1}(p, \varepsilon)$, we observe also that the only case where the maximum of the $\lambda_{i}$ 's is not bounded is when each point $a_{i}$ of the boundary is close to a critical point $z_{j_{i}}$ of $K_{1}$ with $(\partial K / \partial \nu)\left(z_{j_{i}}\right)>0$ and $z_{j_{i}} \neq z_{j_{k}}$ for $i \neq k$, and each point $a_{i}$ in $S_{+}^{4}$ is close to a critical point $y_{j_{i}}$ of $K$ with $j_{i} \neq j_{k}$ for $i \neq k$ and $\rho\left(y_{j_{1}}, \ldots, y_{j_{s}}\right)>0$, where $s=p-q$ and $\rho\left(y_{j_{1}}, \ldots, y_{j_{s}}\right)$ denotes the least eigenvalue of $M\left(y_{j_{1}}, \ldots, y_{j_{s}}\right)$. Hence our proposition follows.

\section{Proof of our results}

Proof of Proposition 1.3. From Proposition 4.2, we know that the only case where the maximum of the $\lambda_{i}$ 's is not bounded is when each point $a_{i}$ of the boundary is close to a critical point $z_{j_{i}}$ of $K_{1}$ with $\partial K / \partial \nu\left(z_{j_{i}}\right)>0$ and $z_{j_{i}} \neq$ $z_{j_{k}}$ for $i \neq k$, and each point $a_{i}$ in $S_{+}^{4}$ is close to a critical point $y_{j_{i}}$ of $K$ with $j_{i} \neq j_{k}$ for $i \neq k$ and $\rho\left(y_{j_{1}}, \ldots, y_{j_{s}}\right)>0$, where $\rho\left(y_{j_{1}}, \ldots, y_{j_{s}}\right)$ denotes the least eigenvalue of $M\left(y_{j_{1}}, \ldots, y_{j_{s}}\right)$ (we remark that all the points can be in the boundary or in $S_{+}^{4}$ ). Arguing as in [3] and [7], we can find a change of variable

$$
\left(a_{1}, \ldots, a_{p}, \lambda_{1}, \ldots, \lambda_{p}\right) \rightarrow\left(\widetilde{a}_{1}, \ldots, \widetilde{a}_{p}, \widetilde{\lambda}_{1}, \ldots, \widetilde{\lambda}_{p}\right):=(\widetilde{a}, \widetilde{\lambda})
$$

such that

$$
\begin{aligned}
J_{0} & \left(\sum_{i=1}^{q} \alpha_{i} \delta_{\left(a_{i}, \lambda_{i}\right)}+\sum_{i=q+1}^{p} \alpha_{i} P \delta_{\left(a_{i}, \lambda_{i}\right)}+\bar{v}\right) \\
= & \frac{\left(S_{4} / 2\right)^{1 / 2}\left(\sum_{i=1}^{q} \alpha_{i}^{2}+2 \sum_{i=q+1}^{p} \alpha_{i}^{2}\right)}{\left(\sum_{i=1}^{q} \alpha_{i}^{4} K\left(\widetilde{a}_{i}\right)+2 \sum_{i=q+1}^{p} \alpha_{i}^{4} K\left(\widetilde{a}_{i}\right)\right)^{1 / 2}} \\
& \times\left[1+A\left((c-\eta) \sum_{i=1}^{q} \frac{1}{K\left(z_{j_{i}}\right)^{2}} \frac{1}{\lambda_{i}} \frac{\partial K}{\partial \nu}\left(z_{j_{i}}\right)+\left(c^{\prime}-\eta\right)^{t} \Lambda M\left(\tau_{s}\right) \Lambda\right)\right]
\end{aligned}
$$

where $c, c^{\prime}$ are two positive constants, $\eta$ is a small positive constant, ${ }^{t} \Lambda=$ $\left(\widetilde{\lambda}_{q+1}^{-1}, \ldots, \tilde{\lambda}_{p}^{-1}\right)$ and $2 A^{-1}=S_{4}\left(\sum_{i=1}^{q} K\left(z_{j_{i}}\right)^{-1}+2 \sum_{i=1}^{s} K\left(y_{j_{i}}\right)^{-1}\right)$.

This yields a spilt of variables $(\alpha, \widetilde{a})$ and $\widetilde{\lambda}$. Thus we deduce that if $(\alpha, \widetilde{a})$ is equal to a critical point, only $\widetilde{\lambda}$ can move. Since $\partial K / \partial \nu\left(z_{j_{i}}\right)>0$ and $\rho\left(y_{j_{1}}, \ldots, y_{j_{s}}\right)>0$, in order to decrease the functional $J_{0}$, we have to increase $\widetilde{\lambda}$, and we obtain a critical point at infinity only in this case. 
We need to compute the Morse index of such a critical point at infinity. For this purpose, we let

$$
f(\alpha)=\left(\sum_{i=1}^{q} \alpha_{i}^{2}+2 \sum_{i=q+1}^{p} \alpha_{i}^{2}\right)\left(\sum_{i=1}^{q} \alpha_{i}^{4} K\left(a_{i}\right)+2 \sum_{i=q+1}^{p} \alpha_{i}^{4} K\left(a_{i}\right)\right)^{-1 / 2} .
$$

$f$ is a homogeneous function, we will show that $\left(1 / K\left(a_{1}\right), \ldots, 1 / K\left(a_{p}\right)\right)$ is a critical point of $f$ and $\tau=\left(\sum_{i=1}^{q} K\left(a_{i}\right)^{-1}+2 \sum_{i=q+1}^{p} K\left(a_{i}\right)^{-1}\right)^{1 / 2}$ is its critical value. In fact, let $\left(1+h_{i}\right)=\alpha_{i}^{2} K\left(a_{i}\right) / \alpha_{p}^{2} K\left(a_{p}\right)$ for $i=1, \ldots, p-1, h_{i}$ goes to 0 and expanding the function around $(0, \ldots, 0) \in \mathbb{R}^{p-1}$, we derive that $(0, \ldots, 0)$ is a nondegenerate maximum point. Finally, using the Morse lemma for $1 / K\left(a_{i}\right)$, the proof of $(1.5)$ follows.

Proof of Theorem 1.4. For $\eta$ small enough, we introduce the following neighborhood of $\Sigma^{+}$

$$
V_{\eta}\left(\Sigma^{+}\right)=\left\{u \in \Sigma / e^{2 J_{0}(u)} J_{0}(u)^{3}\left|u^{-}\right|_{L^{4}}^{2}<\eta\right\}, \quad \text { where } u^{-}=\max (0,-u) .
$$

Our global vector field $Z$ will be built using a convex combination of the vector field $W$ defined in $V(p, \varepsilon)$ for $p \geq 1$ and the vector $Y=-\nabla J_{0}$ outside $\cup_{p \geq 1} V(p, \varepsilon / 2) . V_{\eta}\left(\Sigma^{+}\right)$is invariant under the flow line generated by $Z$ (see [7]). Since $V_{\eta}\left(\Sigma^{+}\right)$is contractible, we have $\chi\left(V_{\eta}\left(\Sigma^{+}\right)\right)=1$, where $\chi$ is the EulerPoincaré characteristic. The proof will be by contradiction, we assume that $J_{0}$ has no critical point in $V_{\eta}\left(\Sigma^{+}\right)$. From Proposition 1.3, we know that the only critical points at infinity of $J_{0}$ in $V_{\eta}\left(\Sigma^{+}\right)$are the family defined by (1.4). Using the vector field $Z$, we have $V_{\eta}\left(\Sigma^{+}\right)$retracts by deformation onto $\cup W_{u}\left(w_{\infty}\right)$ (see sections 7 and 8 of [6]), where $W_{u}\left(w_{\infty}\right)$ is the unstable manifold at infinity of the critical point at infinity $w_{\infty}$. We observe that there are three kind of critical points at infinity $w_{\infty}$ : on the boundary $(s=0)$, in the interior $(q=0)$ and the mixed-type where $q \neq 0$ and $s \neq 0$ where $q$ and $s$ are defined in Proposition 1.3. Let $\Gamma_{q}:=\left\{\left(z_{j_{1}}, \ldots, z_{j_{q}}\right): \nabla K_{1}\left(z_{j_{i}}\right)=0, \partial_{\nu} K\left(z_{j_{i}}\right)>0\right.$ and $z_{j_{i}} \neq z_{j_{k}}$ if $\left.i \neq k\right\}$, where $\partial_{\nu}$ denote the normal derivative of $K$. Then, we have

$$
\begin{aligned}
1= & \sum_{q=1}^{r_{1}} \sum_{\left(j_{1}, \ldots, j_{q}\right) \in \Gamma_{q}}(-1)^{4 q-1-\sum_{j=1}^{q} k_{1}^{j_{i}}}+\sum_{s=1}^{r_{2}} \sum_{\tau_{s}=\left(j_{1}, \ldots, j_{s}\right) / \rho\left(\tau_{s}\right)>0}(-1)^{5 s-1-\sum_{i=1}^{s} k_{j_{i}}} \\
& +\sum_{1 \leq q \leq r_{1}, 1 \leq s \leq r_{2}} \sum_{\Gamma_{q}, \rho\left(\tau_{s}\right)>0}(-1)^{5 s+4 q-1-\sum_{i=1}^{q} k_{1}^{j_{i}}-\sum_{i=1}^{s} k_{j_{i}}}
\end{aligned}
$$

where $k_{1}^{j_{i}}=\operatorname{index}\left(K_{1}, z_{j_{i}}\right)$ and $k_{j_{i}}=\operatorname{index}(K, y)$.

Note that the assumption $\left(H_{5}\right)$ implies that $4 q-\sum_{i=1}^{q} k_{1}^{j_{i}}$ is even for all $q$. Thus

$$
(C+1)\left(1-\sum_{s=1}^{r_{2}} \sum_{\tau_{s}=\left(j_{1}, \ldots, j_{s}\right) / \rho\left(\tau_{s}\right)>0}(-1)^{5 s-1-\sum_{i=1}^{s} k_{j_{i}}}\right)=0 \text {, where } C=\sharp\left(\cup_{q} \Gamma_{q}\right)
$$

which is a contradiction with the assumption of our theorem. Thus there exists at least a critical point of $J_{0}$ in $V_{\eta}\left(\Sigma^{+}\right)$. Arguing as in [7], we prove that this critical point is positive. 
Proof of Theorem 1.6. For the first condition, the existence is proved in [8].

Thus we focus in the case where the second condition holds. Since the assumption (1.7) is satisfied for $y_{0}$, we derive that, $P \delta_{\left(y_{0}, \infty\right)}$ is not a critical point at infinity. In addition, using the fact that $K_{1}\left(z_{0}\right)=\max K_{1}$ and $K\left(y_{0}\right) \geq 2 K_{1}\left(z_{0}\right)$, from Proposition 4.2 , we deduce that all the levels of the critical points at infinity are above $\overline{\mu_{0}}:=\left(S_{4} / K\left(y_{0}\right)\right)^{1 / 2}$. Furthermore, using the expansion of $J_{0}$ in Proposition 7.1 and the assumption (1.7), we have for $\lambda_{0}$ large enough $J_{0}\left(P \delta_{\left(y_{0}, \lambda_{0}\right)}\right) \leq \overline{\mu_{0}}\left(1-c \lambda_{0}^{-2}\right)$. From another part, using again Proposition 7.1, we derive that $J_{0}(u) \geq \overline{\mu_{0}}\left(1-c^{\prime} \varepsilon\right)$, for each $u \in V(p, \varepsilon), p \geq 1$. Thus, for a fixed $\lambda_{0}$ we can choose $\varepsilon$ such that

$$
J_{0}(u)>J_{0}\left(P \delta_{\left(y_{0}, \lambda_{0}\right)}\right), \quad \text { for each } u \in V(p, \varepsilon), \forall p \geq 1 .
$$

Arguing by contradiction and assuming that, there is no critical point of $J_{0}$ under the level $\overline{\mu_{0}}$.

Let $u(s)$ be the solution of the equation (4.1) with $u_{0}=P \delta_{\left(y_{0}, \lambda_{0}\right)} /\left\|P \delta_{\left(y_{0}, \lambda_{0}\right)}\right\|$. From (5.1), we observe that $u(s) \notin V(p, \varepsilon)$, for each $p \geq 1$. Thus, for each $s \geq 0$, we have $(\partial / \partial s) J_{0}(u(s))=-\left|\nabla J_{0}(u(s))\right|^{2} \leq-c^{2}$. This implies that $J_{0}(u(s))$ goes to $-\infty$ as $s \rightarrow+\infty$, which is a contradiction. Hence, the result follows.

\section{Appendix 1}

In this appendix, we collect some expansions of the gradient of the functional $I_{\varepsilon}$ associated to the problem $\left(P_{\varepsilon}\right)$ for $\varepsilon>0$ which are needed in Sect. 2. We start by giving the following lemma which corresponds to [23] and Lemma 2.1 of [10]. (Note that in [23], the Claim is proved for the dimension 3, but the same argument is available in our case).

Lemma 6.1. Let $\delta_{(a, \lambda)}$ be defined in (1.1) and $d=d\left(a, \partial S_{+}^{4}\right)$. For $\varepsilon>0$, we have

(i) $\delta_{(a, \lambda)}^{-\varepsilon}(x)=1-\varepsilon \log \delta_{(a, \lambda)}+O\left(\varepsilon^{2} \log ^{2} \lambda\right)$ in $S_{+}^{4}$.

(ii) For $a \in S_{+}^{4}$, we have $P \delta_{(a, \lambda)}-\delta_{(a, \lambda)}=c_{0} \frac{H(a, .)}{\lambda}+f_{(a, \lambda)}$ where $f_{(a, \lambda)}$ satisfies :

$$
\left|f_{(a, \lambda)}\right|_{L \infty} \leq \frac{c}{\lambda^{3} d^{4}} \quad \text { and } \quad \lambda \frac{\partial f}{\partial \lambda}=O\left(\frac{1}{\lambda^{3} d^{4}}\right) .
$$

Now, explicit computations, using (1.1), Lemma 6.1 and the properties of $M_{\varepsilon}$, yield the following:

Proposition 6.2. For $u=\alpha_{1} \delta_{\left(x_{1}, \lambda_{1}\right)}+\alpha_{2} P \delta_{\left(x_{2}, \lambda_{2}\right)}+v$, with $(\alpha, \lambda, x, v) \in M_{\varepsilon}$, we have:

$$
\begin{aligned}
\left\langle\nabla I_{\varepsilon}(u), \delta_{1}\right\rangle= & \frac{\alpha_{1} S_{4}}{2}\left(1-\alpha_{1}^{2-\varepsilon} K\left(x_{1}\right)\right)+O\left(\varepsilon \log \lambda_{1}+\frac{1}{\lambda_{1}}+\varepsilon_{12}+\|v\|^{2}\right), \\
\left\langle\nabla I_{\varepsilon}(u), \lambda_{1} \frac{\partial \delta_{1}}{\partial \lambda_{1}}\right\rangle= & \alpha_{2}\left(1-\alpha_{2}^{2-\varepsilon} K\left(x_{2}\right)-\alpha_{1}^{2-\varepsilon} K\left(x_{1}\right)\right) c_{2} \lambda_{1} \frac{\partial \varepsilon_{12}}{\partial \lambda_{1}} \\
& +\alpha_{1}^{3-\varepsilon} \frac{\varepsilon S_{4} K\left(x_{1}\right)}{8}
\end{aligned}
$$




$$
\begin{aligned}
+ & \alpha_{1}^{3-\varepsilon} \frac{2 c_{3}}{\lambda_{1}} \frac{\partial K}{\partial \nu}\left(x_{1}\right)+O\left(\|v\|^{2}+\frac{1}{\lambda_{1}^{2}}+\varepsilon^{2} \log \lambda_{1}+\frac{\varepsilon \log \lambda_{1}}{\lambda_{1}}\right) \\
+ & O\left(\varepsilon_{12}^{2} \log \varepsilon_{12}^{-1}+\frac{\varepsilon_{12}}{\lambda_{2}}\left(\log \varepsilon_{12}^{-1}\right)^{1 / 2}\right) \\
\left\langle\nabla I_{\varepsilon}(u), \frac{1}{\lambda_{1}} \frac{\partial \delta_{1}}{\partial x_{1}}\right\rangle= & \left(\alpha_{1} c_{4}\left(1-\alpha_{1}^{2-\varepsilon} K\left(x_{1}\right)\right)+\alpha_{1}^{3-\varepsilon} K\left(x_{1}\right) \varepsilon\left(c_{4} \log \lambda_{1}+c_{7}\right)\right. \\
& \left.+2 \alpha_{1}^{3-\varepsilon} \frac{c_{5}}{\lambda_{1}} \frac{\partial K}{\partial \nu}\left(x_{1}\right)\right) e_{4}+\alpha_{2}\left(1-\sum \alpha_{i}^{2-\varepsilon} K\left(x_{i}\right)\right) \frac{c_{2}}{\lambda_{1}} \frac{\partial \varepsilon_{12}}{\partial x_{1}} \\
& -2 \alpha_{1}^{3-\varepsilon} c_{5} \frac{\nabla_{T} K\left(x_{1}\right)}{\lambda_{1}}+O\left(\|v\|^{2}+\lambda_{2}\left|x_{1}-x_{2}\right| \varepsilon_{12}^{\frac{5}{2}}\right) \\
& +O\left(\varepsilon_{12}^{2} \log \varepsilon_{12}^{-1}+\frac{\varepsilon_{12}}{\lambda_{2}}\left(\log \varepsilon_{12}^{-1}\right)^{\frac{1}{2}}+\frac{1}{\lambda_{1}^{2}}+\varepsilon^{2} \log ^{2} \lambda_{1}\right),
\end{aligned}
$$

where

$$
\begin{gathered}
S_{4}=64 \int_{\mathbb{R}^{4}} \frac{d x}{\left(1+|x|^{2}\right)^{4}}, \quad c_{2}=64 \int_{\mathbb{R}^{4}} \frac{d x}{\left(1+|x|^{2}\right)^{3}}, \quad c_{3}=64 \int_{\mathbb{R}_{+}^{4}} \frac{x_{4}\left(|x|^{2}-1\right)}{\left(1+|x|^{2}\right)^{5}} d x \\
c_{4}=132 \int_{\mathbb{R}_{+}^{4}} \frac{x_{4}}{\left(1+|x|^{2}\right)^{5}} d x, \quad c_{5}=16 \int_{\mathbb{R}^{4}} \frac{x_{4}^{2}}{\left(1+|x|^{2}\right)^{5}} d x .
\end{gathered}
$$

Proposition 6.3. For $u=\alpha_{1} \delta_{\left(x_{1}, \lambda_{1}\right)}+\alpha_{2} P \delta_{\left(x_{2}, \lambda_{2}\right)}+v$, with $(\alpha, \lambda, x, v) \in M_{\varepsilon}$, we have:

$$
\begin{aligned}
\left\langle\nabla I_{\varepsilon}(u), P \delta_{2}\right\rangle= & \alpha_{2} S_{4}\left(1-\alpha_{2}^{2-\varepsilon} K\left(x_{2}\right)\right)+O\left(\varepsilon \log \lambda_{2}+\frac{1}{\lambda_{2}^{2}}+\varepsilon_{12}+\|v\|^{2}\right), \\
\left\langle\nabla I_{\varepsilon}(u), \lambda_{2} \frac{\partial P \delta_{2}}{\partial \lambda_{2}}\right\rangle= & \alpha_{1}\left(1-\sum \alpha_{i}^{2-\varepsilon} K\left(x_{i}\right)\right) c_{2} \lambda_{2} \frac{\partial \varepsilon_{12}}{\partial \lambda_{2}}+\alpha_{2}^{3-\varepsilon} \frac{\varepsilon S_{4} K\left(x_{2}\right)}{4} \\
& +\alpha_{2}^{3-\varepsilon} \frac{c_{2}}{12} \frac{\Delta K\left(x_{2}\right)}{\lambda_{2}^{2}}+\alpha_{2} c_{2} \frac{H\left(x_{2}, x_{2}\right)}{\lambda_{2}^{2}}\left(2 \alpha_{2}^{2-\varepsilon} K\left(x_{2}\right)-1\right) \\
& +O\left(\varepsilon^{2} \log \lambda_{2}+\frac{1}{\lambda_{2}^{3}}+\|v\|^{2}+\varepsilon_{12}^{2} \log \varepsilon_{12}^{-1}\right. \\
& \left.+\frac{1}{\lambda_{2}} \varepsilon_{12}\left(\log \varepsilon_{12}^{-1}\right)^{\frac{1}{2}}\right), \\
\left\langle\nabla I_{\varepsilon}(u), \frac{1}{\lambda_{2}} \frac{\partial P \delta_{2}}{\partial x_{2}}\right\rangle= & \alpha_{1}\left(1-\sum \alpha_{i}^{2-\varepsilon} K\left(x_{i}\right)\right) c_{2} \frac{1}{\lambda_{2}} \frac{\partial \varepsilon_{12}}{\partial x_{2}}-\alpha_{2}^{3-\varepsilon} c_{8} \frac{\nabla K\left(x_{2}\right)}{\lambda_{2}} \\
& +O\left(\frac{\varepsilon \log \lambda_{2}}{\lambda_{2}}\left|\nabla K\left(x_{2}\right)\right|+\frac{1}{\lambda_{2}^{2}}+\|v\|^{2}+\lambda_{1}\left|x_{1}-x_{2}\right| \varepsilon_{12}^{\frac{5}{2}}\right) \\
& +O\left(\varepsilon \varepsilon_{12}\left(\log \varepsilon_{12}^{-1}\right)^{\frac{1}{2}}+\varepsilon_{12}^{2} \log \varepsilon_{12}^{-1}+\frac{\varepsilon_{12}}{\lambda_{1}}\left(\log \varepsilon_{12}^{-1}\right)^{\frac{1}{2}}\right) .
\end{aligned}
$$

\section{Appendix 2}

We are going to give the expansion of the Euler- Lagrange functional $J_{0}$ associated to the problem $(P)$ and its gradient in $V(p, \varepsilon)$ which are useful in the construction of the pseudo gradient. The following propositions are extracted 
from [9] and [10]. The first computations are given in [2]. Note that the constants in this paper seem to be different than [10], but taking account the difference of the notations and the definition of $\delta_{(a, \lambda)}$, we derive that the constants are the same.

Proposition 7.1. For $u=\sum_{i=1}^{q} \alpha_{i} \delta_{\left(a_{i}, \lambda_{i}\right)}+\sum_{i=q+1}^{p} \alpha_{i} P \delta_{\left(a_{i}, \lambda_{i}\right)} \in V(p, \varepsilon)$, we have

$$
\begin{aligned}
J_{0}(u)= & \frac{\left(S_{4} / 2\right)^{1 / 2}\left(\sum_{i=1}^{q} \alpha_{i}^{2}+2 \sum_{i=q+1}^{p} \alpha_{i}^{2}\right)}{\left(\sum_{i=1}^{q} \alpha_{i}^{4} K\left(a_{i}\right)+2 \sum_{i=q+1}^{p} \alpha_{i}^{4} K\left(a_{i}\right)\right)^{\frac{1}{2}}}\left[1+\frac{c_{1}}{\theta} \sum_{i=1}^{q} \frac{1}{K\left(a_{i}\right)^{2}} \frac{1}{\lambda_{i}} \frac{\partial K_{1}}{\partial \nu}\left(a_{i}\right)\right. \\
& -\frac{c_{2}}{2 \theta} \sum_{i \neq j} \frac{\gamma_{i j} \varepsilon_{i j}}{\left(K\left(a_{i}\right) K\left(a_{j}\right)\right)^{1 / 2}}+\frac{c_{2}}{2 \theta} \sum_{i>q}\left(\frac{-\Delta K\left(a_{i}\right)}{3 \lambda_{i}^{2} K\left(a_{i}\right)^{2}}-\frac{4 H\left(a_{i}, a_{i}\right)}{\lambda_{i}^{2} K\left(a_{i}\right)}\right) \\
& \left.-\frac{c_{2}}{2 \theta} \sum_{i, j>q, j \neq i} \frac{4}{\left(K\left(a_{i}\right) K\left(a_{j}\right)\right)^{\frac{1}{2}}} \frac{H\left(a_{i}, a_{j}\right)}{\lambda_{i} \lambda_{j}}\right] \\
& +o\left(\sum_{r \neq k} \varepsilon_{k r}+\sum_{i \leq q} \frac{1}{\lambda_{i}}+\sum_{i>q} \frac{1}{\left(\lambda_{i} d_{i}\right)^{2}}\right),
\end{aligned}
$$

where $S_{4}$ is defined in Proposition $6.2 ; \gamma_{i j}=1$ if $i, j \leq q ; \gamma_{i j}=2$ if not and

$$
c_{1}=64 \int_{\mathbb{R}_{+}^{4}} \frac{x_{4} d x}{\left(1+|x|^{2}\right)^{4}}, \quad \theta=\frac{S_{4}}{2}\left(\sum_{i \leq q} K\left(a_{i}\right)^{-1}+2 \sum_{i>q} K\left(a_{i}\right)^{-1}\right) .
$$

Proposition 7.2. For $u=\sum_{i=1}^{q} \alpha_{i} \delta_{\left(a_{i}, \lambda_{i}\right)}+\sum_{i=q+1}^{p} \alpha_{i} P \delta_{\left(a_{i}, \lambda_{i}\right)} \in V(p, \varepsilon)$, we have:

$$
\begin{aligned}
\left\langle\nabla J_{0}(u), \lambda_{i} \frac{\partial \delta_{i}}{\partial \lambda_{i}}\right\rangle= & -c_{2} J_{0}(u) \sum_{j \leq q, j \neq i} \alpha_{j} \lambda_{i} \frac{\partial \varepsilon_{i j}}{\partial \lambda_{i}}-4 c_{3} J_{0}(u)^{3} \frac{\alpha_{i}^{3}}{\lambda_{i}} \frac{\partial K}{\partial \nu}\left(a_{i}\right) \\
& +O\left(\sum_{i \leq q, j>q} \varepsilon_{i j}+\sum_{k, r \leq q} \varepsilon_{k r}^{2}+\sum_{i \leq q} \frac{1}{\lambda_{i}^{2}}\right) \\
\left\langle\nabla J_{0}(u), \frac{1}{\lambda_{i}} \frac{\partial \delta_{i}}{\partial a_{i}}\right\rangle= & 2 \alpha_{i} J_{0}(u) e_{4}\left(c_{4}\left(1-J_{0}(u)^{2} \alpha_{i}^{2} K\left(a_{i}\right)\right)+c_{5} J_{0}(u)^{2} \frac{\alpha_{i}^{2}}{\lambda_{i}} \frac{\partial K}{\partial \nu}\left(a_{i}\right)\right) \\
& -8 c_{5} J_{0}(u)^{3} \frac{\alpha_{i}^{3}}{\lambda_{i}} \nabla_{T} K\left(a_{i}\right)-c_{2} J_{0}(u) \sum_{j \leq q, j \neq i} \frac{\alpha_{j}}{\lambda_{i}} \frac{\partial \varepsilon_{i j}}{\partial a_{i}}(1+o(1)) \\
& +O\left(\sum_{i \leq q, j>q} \varepsilon_{i j}+\sum_{k \leq q} \lambda_{k}\left|a_{i}-a_{k}\right| \varepsilon_{k r}^{5 / 2}+\sum_{i \leq q} \frac{1}{\lambda_{i}^{2}}\right), \\
\left\langle\nabla J_{0}(u), \delta_{i}\right\rangle= & \alpha_{i} J_{0}(u) S_{4}\left(1-J_{0}(u)^{2} \alpha_{i}^{2} K\left(a_{i}\right)\right)+O\left(\frac{1}{\lambda_{i}}+\sum \varepsilon_{i j}\right),
\end{aligned}
$$

where $\left(e_{1}, \ldots, e_{4}\right)$ denotes an orthogonal basis of $T_{a_{i}} S_{+}^{4}$ with $\left\|e_{i}\right\|=1$. 
Proposition 7.3. For $u=\sum_{i=1}^{q} \alpha_{i} \delta_{\left(a_{i}, \lambda_{i}\right)}+\sum_{i=q+1}^{p} \alpha_{i} P \delta_{\left(a_{i}, \lambda_{i}\right)} \in V(p, \varepsilon)$, we have:

$$
\begin{aligned}
\left\langle\nabla J_{0}(u), \lambda_{i} \frac{\partial P \delta_{i}}{\partial \lambda_{i}}\right\rangle= & c_{2} J_{0}(u)\left(-2 \sum_{j \neq i} \alpha_{j} \lambda_{i} \frac{\partial \varepsilon_{i j}}{\partial \lambda_{i}}+4 \sum_{j=q+1, j \neq i}^{p} \alpha_{j} \frac{H\left(a_{i}, a_{j}\right)}{\lambda_{i} \lambda_{j}}\right. \\
& \left.+\alpha_{i} \frac{\Delta K\left(a_{i}\right)}{3 \lambda_{i}^{2} K\left(a_{i}\right)}+4 \alpha_{i} \frac{H\left(a_{i}, a_{i}\right)}{\lambda_{i}^{2}}\right) \\
& +o\left(\sum_{i \neq j} \varepsilon_{i j}+\sum_{i>q} \frac{1}{\left(\lambda_{i} d_{i}\right)^{2}}\right), \\
\left\langle\nabla J_{0}(u), \frac{1}{\lambda_{i}} \frac{\partial P \delta_{i}}{\partial a_{i}}\right\rangle & . \nabla \\
\hline & K\left(a_{i}\right) \geq c \frac{\left|\nabla K\left(a_{i}\right)\right|^{2}}{\lambda_{i}} \\
& +O\left(\left(\frac{1}{\left(\lambda_{i} d_{i}\right)^{2}}+\sum_{i \neq j} \varepsilon_{i j}\right)\left|\nabla K\left(a_{i}\right)\right|\right) .
\end{aligned}
$$

\section{References}

[1] Ambrosetti, A., Li, Y.Y., Malchiodi, A.: Yamabe and Scalar Curvature Problems under boundary conditions, preprint S.I.S.S.A., ref. 52/2000/M. preliminary note on C.R.A.S. Série 1, vol. 330, pp. 1013-1018 (1989)

[2] Bahri, A.: Critical point at infinity in some variational problems. Pitman Research Notes in Mathematics Series, vol. 182. Longman Sci. Tech., Harlow (1989)

[3] Bahri, A.: An invarient for Yamabe-type flows with applications to scalar curvature problems in high dimension. A celebration of J. F. Nash Jr.. Duke Math. J. 81, 323-466 (1996)

[4] Bahri, A., Coron, J.M.: The scalar curvature problem on the standard three dimensional spheres. J. Funct. Anal. 95, 106-172 (1991)

[5] Bahri, A., Li, YY., Rey, O.: On a variational problem with lack of compactness: the topological effect of the critical points at infinity. Calc. Var Partial Differ. Equ. 3, 67-94 (1995)

[6] Bahri, A., Rabinowitz, P.: Periodic orbits of hamiltonian systems of three body type. eAnn. Inst. H. Poincaré Anal. Non lináire 8, 561-649 (1991)

[7] Ben Ayed, M., Chen, Y., Chtioui, H., Hammami, M.: On the prescribed scalar curvature problem on 4-manifolds. Duke Math. J. 84, 633-677 (1996)

[8] Ben Ayed, Chtioui, H.: Topological tools in prescribing the scalar curvature on the half sphere. Adv. Nonlinear Stud. 4, 121-148 (2004) 
[9] Ben Ayed, M., El Mehdi, K., Ould Ahmedou, M.: Prescribing the scalar curvature under minimal boundary condition on the half sphere. Adv. Nonlinear Stud. 2, 93-116 (2002)

[10] Ben Ayed, M., El Mehdi, K., Ould Ahmedou, M.: The scalar curvature problem on the four dimensional half sphere. Calc. Var. 22, 465-482 (2005)

[11] Bianchi, G., Pan, X.B.: Yamabe equations on half spheres. Nonlinear Anal. 37, 161-186 (1999)

[12] Brezis, H., Coron, J.M.: Convergence of solutions of H-systems or how to blow bubbles. Arch. Ration. Mech. Anal. 89, 21-56 (1985)

[13] Chang, S.A., Yang, P.: A perturbation result in prescribing scalar curvature on $S^{n}$. Duke Math. J. 64, 27-69 (1991)

[14] Cherrier, P.: Problèmes de Neumann non linéaires sur les variétés Riemaniennes. J. Funct. Anal. 57, 154-207 (1984)

[15] Djadli, Z., Malchiodi, A., Ould Ahmedou, M.: Prescribing the scalar and the boundary mean curvature on the three dimensional half sphere. J. Geom. Anal. 13, 233-267 (2003)

[16] Escobar, J.: Conformal deformation of Riemannian metric to scalar flat metric with constant mean curvature on the boundary. Ann. Math. 136, 1-50 (1992)

[17] Escobar, J.: Conformal metrics with prescribed mean curvature on the boundary. Calc. Var. 4, 559-592 (1996)

[18] Han, Z.C., Li, Y.Y.: The existence of conformal metrics with constant scalar curvature and constant boundary mean curvature. Commun. Anal. Geom. 8, 809$869(2000)$

[19] Li, Y.Y.: Prescribing scalar curvature on $S^{n}$ and related topics, Part I. J. Differ. Equ. 120, 319-410 (1995)

[20] Li, Y.Y.: Part II, existence and compactness. Commun. Pure Appl. Math. 49, 437-477 (1996)

[21] Li, Y.Y.: The Niremberg problem in a domain with boundary. Top. Methods Nonlinear Anal. 6, 309-329 (1995)

[22] Lions P.L.: The concentration compactness principle in the calculus of variations. The limit case. Rev. Mat. Iberoamericana 1, I: 165-201; II: 45-121 (1985)

[23] Rey, O.: The topological impact of critical points at infinity in a variational problem with lack of compactness: the dimension 3. Adv. Differ. Equ. 4, 581616 (1999)

[24] Struwe, M.: A global compactness result for elliptic boundary value problems involving nonlinearities. Math. Z. 187, 511-517 (1984) 
Mohamed Ben Ayed and Kamal Ould Bouh

Département de Mathématiques

Faculté des Sciences

Université de Sfax

Sfax

Tunisia

e-mail: Mohamed.Benayed@fss.rnu.tn

Kamal Ould Bouh

e-mail: kamal_bouh@yahoo.fr

Rabeh Ghoudi

Département de Mathématiques

Faculté des Sciences

Université de Gabès

Gabès

Tunisia

e-mail: ghoudi.rabeh@yahoo.fr

Received: 24 March 2010.

Accepted: 3 December 2011. 